\title{
The Double Absorbing Boundary Method for a Class of Anisotropic Elastic Media
}

\author{
Daniel Rabinovich ${ }^{(1) *}$ \\ Jacobo Bielak ${ }^{(2) \ddagger}$
}

\author{
Dan Givoli ${ }^{(1) \dagger}$ \\ Thomas Hagstrom ${ }^{(3) \S}$
}

(1) Department of Aerospace Engineering, Technion - Israel Institute of Technology, Haifa 32000, Israel

(2) Department of Civil and Environmental Engineering, Carnegie Mellon University, Pittsburgh, PA 15213, U.S.A.

(3) Department of Mathematics, Southern Methodist University, Dallas, TX 75275, U.S.A.

\author{
REVISED
}

October 23, 2016

\footnotetext{
*E-mail: aedaniel@technion.ac.il

${ }^{\dagger}$ Corresponding author.

E-mail: givolid@technion.ac.il, Tel.: +1-972-829-3814, Fax: +1-972-829-2030.

Currently on leave at the Faculty of Civil Engineering \& Geosciences, Technical University of Delft, Delft, The Netherlands.

‡E-mail: jbielak@cmu.edu

$\S$ E-mail: thagstrom@smu.edu
} 


\section{Abstract}

The Double Absorbing Boundary (DAB) method was recently introduced as a new approach for solving wave problems in unbounded domains. It has common features to local highorder Absorbing Boundary Conditions (ABC) on one hand, and to Perfectly Matched Layers (PML) on the other, and has relative advantages with respect to both. In the DAB method, the unbounded domain is truncated to produce a finite computational domain, which is then enclosed by a thin layer, as in the PML. Local high-order ABC are then imposed on both the inner and outer boundaries of the layer. Auxiliary variables are defined on the two boundaries as well as inside the layer, and participate in the numerical scheme. Only low order derivatives appear in the formulation, which allows the use of standard discretization methods in space and time. In previous studies, the DAB was developed for acoustic waves which are solutions to the scalar wave equation, and for elastic waves in isotropic media. Here the approach is extended to time-dependent elastic waves in anisotropic media. The geometrical configuration assumed is that of a semi-infinite wave guide, truncated via the DAB layer. Standard Finite Elements (FE) are used for space discretization and the damped Newmark scheme is used for time discretization. The problems considered here are restricted to those with periodic lateral boundary conditions, and with anisotropic media which do not support inverse modes, since removing these limitations may lead to a numerical instability. The performance of the scheme is demonstrated via numerical examples, including uniform orthotropic and layered orthotropic media.

Keywords: Double absorbing boundary, Absorbing boundary condition, High-order, Auxiliary variables, Elastic waves, Anisotropic, Elastodynamics, Finite elements. 


\section{Introduction}

About two decades ago, a revolution occurred in the area of computational methods for wave problems in exterior media, when two approaches were invented for the high fidelity solution of unbounded domain problems: the use of high-order Absorbing Boundary Conditions $(\mathrm{ABC})$ and the Perfectly Matched Layer (PML). Research on these methods remains very active. See, e.g., the review papers [1-4]. The interest in these methods is common to various fields of application, such as acoustics, geophysics and electro-magnetic waves. It turns out that in some cases it is very difficult to find an absorbing boundary scheme, as it is often called, that is at the same time stable, sufficiently accurate, computationally efficient, robust, and can be employed in conjunction with standard interior computational schemes.

The method of high-order ABCs was devised by Collino [5] in 1993. High-order ABCs are local in space and time, like the classical ABCs of Engquist and Majda [6] and Bayliss and Turkel [7], but unlike those, they do not involve high-order derivatives. Therefore they can be implemented in practice up to any desired order, as opposed to the classical ones that have been implemented up to second order only. In the high-order $\mathrm{ABC}$ scheme, the order of the $\mathrm{ABC}$ is simply an input parameter. The high derivatives that initially appear when designing a high-order ABC are eliminated by introducing auxiliary variables $\phi_{j}$ on the boundary. Different formulations with the same basic idea followed that of Collino; see, e.g., references in the review paper [2].

The PML method was invented by Bérenger [8] in 1994 for electromagnetic waves. Since then it has been further developed, analyzed and used in various applications by many authors. See, e.g., references in the review paper [4]. The PML is a layer adjacent to the boundary that truncates the unbounded domain, in which the governing equations are artificially modified. It possesses two properties at the continuous level: (a) there is a perfect match between the layer and the interior domain, namely introducing the (unbounded) PML does not change the solution in the computational domain so long as all data/sources are localized there [9]; and (b) the solution decays exponentially when it travels inside the layer. These two properties theoretically guarantee excellent performance of the PML. What may sometimes hamper this theoretical performance is the sensitivity of the PML to the discretization and the need to introduce ad-hoc damping and stretching profiles.

Each of the two classes of techniques has relative advantages. One disadvantage of highorder $\mathrm{ABCs}$ is that they require special treatment at corners formed by the intersection of two flat segments of the artificial boundary, and in some cases also at corners between an artificial and a physical boundary. Such special treatment is sometimes cumbersome or even difficult to devise. In contrast, handling corners with PMLs is usually straight forward. Another disadvantage of high-order ABCs is that they are constrained not to include any normal derivative of an auxiliary variable $\phi_{j}$ on the boundary, since the $\phi_{j}$ are discretized in practice only on the boundary. Thus, the $\mathrm{ABC}$ is allowed to involve only tangential and temporal derivatives of the $\phi_{j}$. Eliminating the normal derivatives from the ABC operators is sometimes difficult and may require a lot of algebra; a case in point is elastodynamics [10]. 
PML is also usually easier to incorporate in an existing numerical code.

On the other hand, an important disadvantage of PML is that it is not associated with a clear notion of convergence, except under the expensive scenario of widening a layer where all physical and auxiliary fields are well-resolved. (In fact, any absorbing boundary scheme can be improved by moving the truncating boundary away towards infinity, with the price of an increasingly high computational effort. The whole point of PML and ABC schemes is to obtain an accurate solution in a small domain.) By contrast, in the case of high-order ABCs, with a fixed location of the boundary, one can approach the exact solution arbitrarily closely (up to discretization error) by increasing the order $P$ of the ABC. Moreover, the computational cost involved increases only linearly with $P$. Also, PMLs seem to be more sensitive to discretization and to the computational parameters than ABCs. A good design of an $\mathrm{ABC}$ at the continuous level usually guarantees good performance at the discrete level. This does not seem to be the general case for PML, where the matching between the solutions in the interior and in the layer at the discrete level is sometimes far from perfect. In addition, the theoretical analysis of a PML is usually more difficult than that for a high-order ABC for the same application. Additional discussion and some quantitative comparisons of the two types of methods can be found in $[1,3,11,12]$.

Recently we presented a new method [13], called the Double Absorbing Boundary (DAB) method, which shares some features of both the PML and the high-order ABC, but enjoys some of the advantages that each of them lacks. In the new method, the computational domain is enclosed by a thin layer, as in the PML scheme. A high order ABC is then imposed on both the inner and outer boundaries of the layer. Auxiliary variables are defined on the two boundaries as well as inside the layer. Like the PML, the DAB does not require special treatment of corners. Also, there is no difficulty in the appearance of normal derivatives of the auxiliary variables in the layer and on its boundaries. This leads to a relatively simple algebra. As in the method of high-order ABCs on a single boundary, the DAB is clearly associated with the notion of convergence; one can approach the exact solution arbitrarily closely (up to discretization error) by increasing the order $P$, with only linearly-increasing cost. The numerical properties of the DAB, like accuracy, stability and sensitivity to discretization, are similar to those of a high-order $\mathrm{ABC}$ on a single boundary. Admittedly, the implementation of PML is still somewhat easier than that of DAB.

In [13], the new method was applied to the scalar wave equation. We incorporated the DAB in a fully explicit finite difference scheme in 1D, and in a Finite Element (FE) scheme in 2D. In [14], a well-posedness proof was provided for the DAB scheme for the acoustics problem written in second-order form. The energy method was employed to obtain uniformin-time estimates of the norm of the solution and the auxiliary functions, thus establishing the well-posedness and asymptotic stability of the DAB formulation. In addition, in [14] the DAB was applied to problems in 2D isotropic elastodynamics, written in first-order conservation form. The problem was discretized using the Lax-Wendroff finite difference scheme. In [15], we applied the method to isotropic elasticity in layered media. Although DAB is a general approach, and in principle can be used with any high-order ABC applied on the double 
boundary, in [13-15] the ABCs of the form proposed by Hagstrom and Warburton (H-W) in $[16,17]$ were considered. The $\mathrm{ABC}$ formulation in [17], called the Complete Radiation Boundary Condition (CRBC), generalizes that in [16], and leads to an almost uniformin-time error estimate for both propagating and decaying waves. Lastly we note that an implementation of DAB for the popular Yee scheme in computational electromagnetism is discussed in [18] and is available in the initial release of the software library rbcpack $^{1}$.

Even before the H-W type ABC was applied to the DAB scheme, it was imposed on a single artificial boundary for problems in elastodynamics [19,20]. This is the first known high-order $\mathrm{ABC}$ for elastodynamics that is long-time stable. The key to obtaining stability turns out to be the use of the Lysmer-Kuhlemeyer (LK) ABC as the termination condition of the recursive relations satisfied by the auxiliary variables $\phi_{j}$. The LK ABC is classical $[21,22]$ and is commonly employed in solid earth geophysics computations, either as originally proposed or with some improvements; see, e.g., [23-25]. In the stable high-order $\mathrm{ABC}$ for elastodynamics, all the recursive relations except the last one are scalar in nature (as in the $\mathrm{H}-\mathrm{W} \mathrm{ABC}$ ), while the last one is the vectorial LK condition. In [19], we have proved this combination to be stable and converging, at the continuous level. The DAB for elastodynamics uses the same recursive relations, except that, in contrast to [19,20], there is no need to eliminate the normal derivatives on the boundaries of the layer.

In this paper, we adapt the DAB method for use for the solution of $2 \mathrm{D}$ elastodynamics problems in anisotropic media. An important application is solid earth geophysics, where the medium is often quite general: heterogeneous, anisotropic and porous. Another motivation may be the understanding of wave phenomena and failure mechanisms in composite materials. We incorporate the DAB in a standard FE scheme, and use a damped Newmark time-stepping to enhance numerical stability.

Our formulation and numerical examples assume periodic boundary conditions along the boundaries perpendicular to the artificial DAB boundaries. This choice is made since some stability issues arise when the periodic conditions are replaced by some physical boundary conditions (e.g., traction free conditions). Attempts to resolve these issues are underway. A similar issue arises with some standard PML formulations, as shown by Duru and Kreiss [26] for the case of an elastic wave-guide with traction-free lateral boundary conditions. By slightly modifying the PML formulation, the authors are able to resume stability. Classical ABCs do not usually exhibit an instability as a result of their interaction with a physical boundary. This is related to the fact that these ABCs involve no auxiliary variables, but only the primary variable, which is defined continuously on all boundaries. For example, the LK condition is stable regardless of the lateral boundary condition.

In solving elastic wave problems (and in fact also acoustic wave problems) in an anisotropic medium, one has to pay attention to the issue of "inverse modes," namely waves associated with a mismatch between the directions of phase and group velocities. Bécache et al. [27] showed that this mismatch plays an important role in the stability analysis of

\footnotetext{
${ }^{1}$ www.rbcpack.org
} 
the PML. In fact, for some cases in anisotropic elasticity the PML becomes unstable due to this mismatch. See also [9] on PML stability. In [28], Meza-Fajardo and Papageorgiou devised a split-PML formulation (M-PML) which is stable in the presence of inverse modes, by introducing damping in both directions of the layer (rather than only in the normal direction). However, this additional damping ruins the analytic continuation induced by the coordinate stretching in the PML, and hence the layer is not perfectly matched anymore. A similar idea was adapted to an unsplit formulation (CPML) by Martin et al. [29]. Most anisotropic elastic media do not support inverse modes, and for them PMLs in open domains are typically stable; see, e.g., [30-33]. However, even for isotropic media, such destabilizing modes do occur in waveguides [34].

Inverse modes appear in anisotropic acoustics too, but, as shown in [35], they can easily be controlled by simple ABC operators. See also [36] on the discrete stability of PML for anisotropic acoustics. Unfortunately, controlling inverse modes is much more difficult in anisotropic elasticity, in those media which support such modes. One can perhaps find some comfort in the fact that such media are very strongly anisotropic and are not so typical in realistic applications. In the present paper we do consider in passing such a medium, but do not resolve the stability issue associated with it.

Following is the outline of the remaining sections. We start Section 2 by a brief review of the equations of anisotropic elastodynamics. Then we derive basic (first-order) ABCs for anisotropic elasticity, of two kinds: a scalar ABC and a vectorial ABC. Both will be used later in the DAB scheme. In Section 3 we discuss the DAB scheme for anisotropic elastic media. In Section 4 we present the FE scheme incorporating this DAB construction. We also discuss some computational aspects of the method. In Section 5 we present numerical examples which demonstrate the performance of the scheme. These include $2 \mathrm{D}$ wave propagation in a uniform orthotropic medium, as well as in a layered orthotropic medium. Note that although the theory is developed for general elastic anisotropy, in this section we specialize the scheme to orthotropic media, which is already representative of the difficulties that may be encountered (e.g., inverse modes). We conclude with some remarks in Section 6.

\section{Basic Absorbing Boundary Conditions for Anisotropic Elastodynamics}

\subsection{The original unbounded-domain problem}

We consider a two-dimensional semi-infinite elastic wave guide of width $b$, as shown in Fig. 1(a). A Cartesian coordinate system $(x, y)$ is introduced with the origin at the southwest corner, so that the waveguide is parallel to the $x$ direction, and $y \in[0, b]$. The south, north and west boundaries of the waveguide are denoted $\Gamma_{S}, \Gamma_{N}$ and $\Gamma_{W}$, respectively. 
(a)

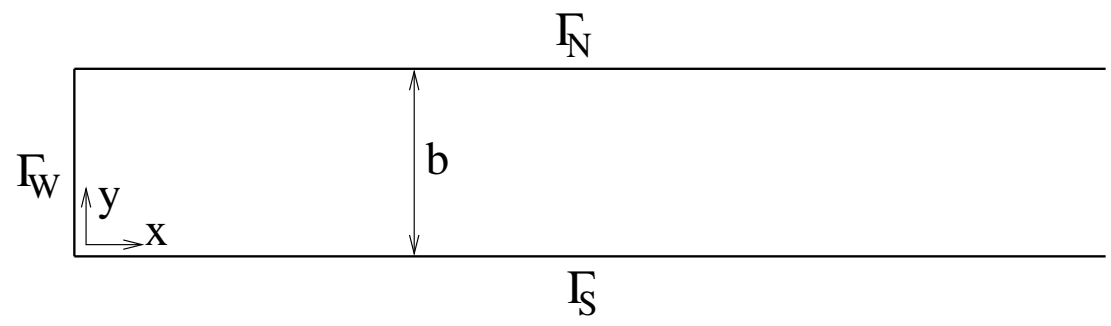

(b)

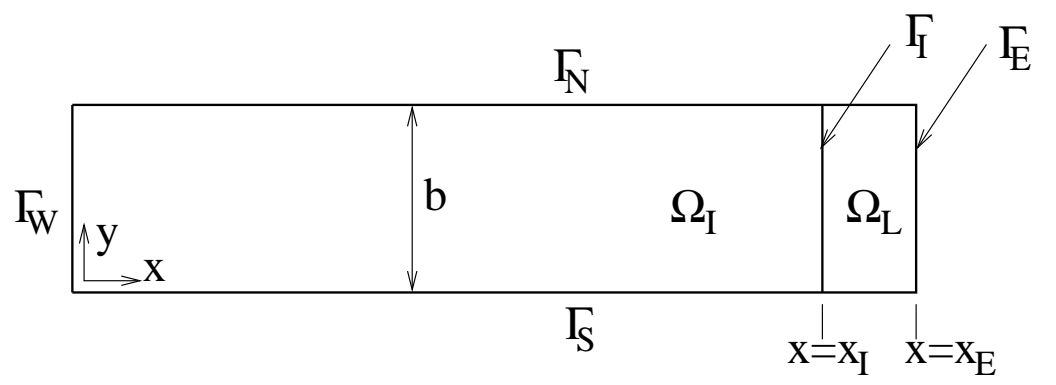

Figure 1: A semi-infinite waveguide: (a) the original setup, (b) the setup of the problem with truncated domain.

In the waveguide we consider the equation of elastodynamics, i.e.,

$$
\rho \ddot{u}_{i}=\sigma_{i j, j}(\boldsymbol{u})+f_{i},
$$

where

$$
\begin{aligned}
\sigma_{i j} & =C_{i j k l} \epsilon_{k l}, \\
\epsilon_{k l} & =\left(u_{k, l}+u_{l, k}\right) / 2 .
\end{aligned}
$$

Here and elsewhere, the summation convention over indices is implied, and a comma subscript denotes partial differentiation with respect to the variable following it. The values $x$ and $y$ are assigned to the indices $i, j, k, l$. A superposed dot indicates differentiation with respect to time. In (1)-(3), $\rho$ is the density of the elastic medium, $\boldsymbol{u}=\left\{u_{i}\right\}$ is the vector field of displacements, $\boldsymbol{\sigma}=\left[\sigma_{i j}\right]$ is the stress tensor, $\boldsymbol{f}=\left\{f_{i}\right\}$ is the vector of applied body-forces, $\boldsymbol{C}=\left[C_{i j k l}\right]$ is the tensor of elastic moduli, and $\boldsymbol{\epsilon}=\left[\epsilon_{i j}\right]$ is the strain tensor.

We shall also define the traction vector acting on a plane with a normal unit vector $\boldsymbol{n}$ as

$$
T_{i}=\sigma_{i j} n_{j}
$$

We employ Voigt notation to reduce the tensor $\boldsymbol{C}=\left[C_{i j k l}\right]$ to the two-dimensional symmetric tensor $\boldsymbol{c}=\left[c_{I J}\right], I, J=1, \ldots, 3$. In this notation, the index pairs $(i, j)$ and $(k, l)$ are transformed to the single indices $I$ and $J$, respectively, according to the rule

$$
(1,1) \rightarrow 1, \quad(2,2) \rightarrow 2, \quad(1,2)=(2,1) \rightarrow 3 .
$$

For the particular case of an orthotropic medium whose principal axes coincide with the $(x, y)$ system, we have $c_{13}=c_{23}=0$, and for the case of an isotropic material, additionally, 
the elastic moduli can be expressed in terms of the Lamé coefficients $\lambda$ and $\mu$, i.e.,

$$
c_{11}=c_{22}=\lambda+2 \mu, \quad c_{12}=\lambda, \quad c_{33}=\mu .
$$

Boundary conditions are specified on the three boundaries. On $\Gamma_{N}$ and $\Gamma_{S}$ we impose periodic boundary conditions:

$$
\begin{aligned}
& u_{i}(x, 0, t)=u_{i}(x, b, t) \\
& u_{i, y}(x, 0, t)=u_{i, y}(x, b, t) .
\end{aligned}
$$

Periodic boundary conditions allow us to detach the study of the stability and accuracy of the $\mathrm{ABC}$, which will be discussed later, from the effects of corners and interactions between the absorbing boundary and other boundaries. We note that (7) and (8) imply the following periodicity condition of the traction:

$$
T_{i}(x, 0, t)=-T_{i}(x, b, t) .
$$

The appearance of the minus sign can be explained by the following calculation:

$$
T_{i}=\sigma_{i j} n_{j}=C_{i j k l} \epsilon_{k l} n_{j}=C_{i j k l} u_{k, l} n_{j} .
$$

From (7) and (8) we deduce that $u_{k, l}$ is periodic. However, the outward normal vector $\boldsymbol{n}$ has an opposite sign on $y=0$ and $y=b$; hence the result (9).

On $\Gamma_{W}$ any boundary condition may be imposed, we choose to prescribe zero displacement boundary conditions

$$
\begin{array}{lll}
u_{x}=0 & \text { on } & \Gamma_{W} \\
u_{y}=0 & \text { on } & \Gamma_{W},
\end{array}
$$

Initial conditions are also given, i.e.,

$$
\begin{aligned}
& u_{i}(x, y, t=0)=u_{i 0}(x, y), \\
& \dot{u}_{i}(x, y, t=0)=\dot{u}_{i 0}(x, y)
\end{aligned}
$$

where $\boldsymbol{u}_{0}=\left\{u_{i 0}\right\}$ and $\dot{\boldsymbol{u}}_{0}=\left\{\dot{u}_{i 0}\right\}$ are known functions. We assume that outside a compact region, denoted $\Omega_{0}$, in which $C_{i j k l}, f_{i}, u_{i 0}$ and $\dot{u}_{i 0}$ may, in principle, be general, the following simplified conditions hold: (a) the medium is homogeneous, namely $C_{i j k l}$ is constant; (b) there are no sources, namely $f_{i}=0$; and (c) the initial values vanish, namely $u_{i 0}=0$ and $\dot{u}_{i 0}=0$.

\subsection{Basics of anisotropic elastodynamics}

We consider traveling waves in the medium described by (1)-(3), which have a frequency of $\omega$ and a wave vector of $\boldsymbol{k}=\left(k_{1}, k_{2}\right)=\left(k_{x}, k_{y}\right)$. These two magnitudes define the components of the phase velocity $V_{i}^{p}$ and the group velocity $V_{i}^{g}$ of the waves:

$$
V_{i}^{p}=\frac{\omega}{k_{i}} ; \quad V_{i}^{g}=\frac{\partial \omega(\boldsymbol{k})}{\partial k_{i}} .
$$


The phase velocity is the velocity at which a particular phase of the wave (for example, a crest) travels, while the group velocity is the velocity at which the energy of the wave propagates in space. These velocities do not necessarily coincide, and in fact their components may have opposite signs. Now, consider a traveling wave that is a solution of (1)-(3), which has the form

$$
\boldsymbol{u}(\boldsymbol{x}, t)=\boldsymbol{D} e^{i(\omega t-\boldsymbol{k} \cdot \boldsymbol{x})}, \quad \boldsymbol{x}=\left(x_{1}, x_{2}\right)=(x, y) .
$$

Here $\boldsymbol{D}$ is the displacement amplitude vector. Consider an artificial boundary, whose outward normal points in the positive $x=x_{1}$ direction. By definition, the wave (16) is an outgoing wave with respect to this boundary if its group velocity (and not necessarily its phase velocity) is directed towards the boundary, i.e., $V_{1}^{g}>0$. If the signs of $V_{1}^{g}$ and $V_{1}^{p}$ are different, we call the wave an "inverse mode". As mentioned in the Introduction, controlling inverse modes by an $\mathrm{ABC}$ (or a PML) is difficult. If we wish to construct an $\mathrm{ABC}$ so that it absorbs outgoing waves, the identification of outgoing and incoming waves must be based on the group velocity rather than on the phase velocity. An ABC based on the phase velocity (as most existing ABCs are) would identify an outgoing inverse mode as incoming, and vice versa. This would give rise to an instability of the scheme. When inverse modes are not present, which is the case in the majority of elastodynamics problems, this difficulty does not arise.

In order for (16) to be a solution of (1)-(3), $\boldsymbol{k}$ and $\omega$ need to satisfy the dispersion relation. This relation is obtained by substituting the wave form (16) in the elastodynamics equations (1)-(3); see, e.g., Landu and Lifshitz [37]. This yields the matrix eigenvalue problem

$$
\boldsymbol{E D}=\omega^{2} \boldsymbol{D}
$$

where the matrix $\boldsymbol{E}$ is the $2 \times 2$, symmetric matrix

$$
E_{i j}=\frac{1}{\rho} C_{i k j l} k_{k} k_{l}
$$

Solving this eigenvalue problem gives (see for example [27])

$$
\left|\begin{array}{ll}
c_{11} k_{x}^{2}+2 c_{13} k_{x} k_{y}+c_{33} k_{y}^{2}-\rho \omega^{2} & c_{13} k_{x}^{2}+\left(c_{12}+c_{33}\right) k_{x} k_{y}+c_{32} k_{y}^{2} \\
c_{13} k_{x}^{2}+\left(c_{12}+c_{33}\right) k_{x} k_{y}+c_{32} k_{y}^{2} & c_{33} k_{x}^{2}+2 c_{23} k_{x} k_{y}+c_{22} k_{y}^{2}-\rho \omega^{2}
\end{array}\right|=0
$$

which can be rewritten as

$$
F=A_{0} k_{x}^{4}+A_{1} k_{x}^{3} k_{y}+A_{2} k_{x}^{2} k_{y}^{2}+A_{3} k_{x} k_{y}^{3}+A_{4} k_{y}^{4}-\left[B_{0} k_{x}^{2}+B_{1} k_{x} k_{y}+B_{2} k_{y}^{2}\right] \rho \omega^{2}+\rho^{2} \omega^{4}=0,
$$






(a)

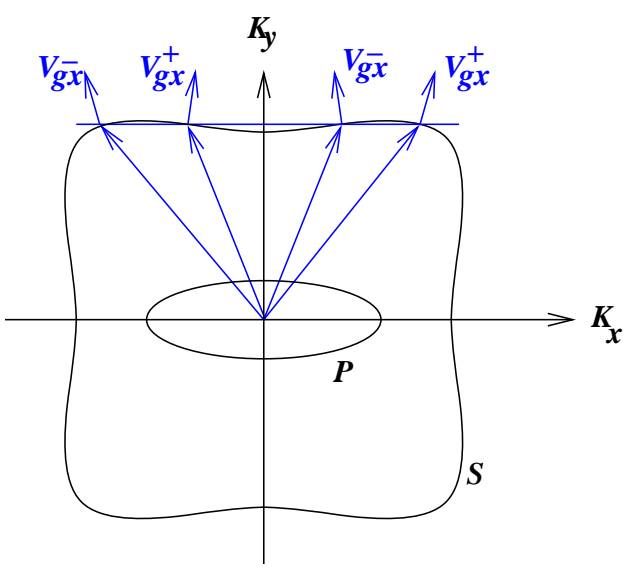

(b)

Figure 2: Slowness diagrams for two anisotropic elastic materials: (a) anisotropy which does not support inverse modes; (b) anisotropy which supports inverse modes.

where

$$
\begin{aligned}
& A_{0}=c_{11} c_{33}-c_{13}^{2} \\
& A_{1}=2\left(c_{11} c_{23}-c_{12} c_{13}\right) \\
& A_{2}=c_{11} c_{22}+2 c_{13} c_{23}-c_{12}^{2}-2 c_{12} c_{33} \\
& A_{3}=2\left(c_{13} c_{22}-c_{12} c_{23}\right) \\
& A_{4}=c_{22} c_{33}-c_{23}^{2} \\
& B_{0}=c_{11}+c_{33} \\
& B_{1}=2\left(c_{13}+c_{23}\right) \\
& B_{2}=c_{22}+c_{33} .
\end{aligned}
$$

Equation (20) can be considered as a fourth-order implicit equation in the components of the slowness vector $\boldsymbol{K}=\left(K_{x}, K_{y}\right)=\left(k_{x} / \omega, k_{y} / \omega\right)$. The curve corresponding to this equation, called the slowness diagram, can be drawn in a system of the coordinate axes $K_{y}$ vs. $K_{x}$. Fig. 2 shows two examples of a slowness diagram for two different anisotropic elastic materials. In each diagram, the radial arrow is in the direction of the phase velocity, while the outward normal to the curve is in the direction of the group velocity. In Fig. 2(a), the $x$ projections of the phase and group velocities have the same sign, over the entire curve, while in Fig. 2(b), parts of the curve are associated with $x$-projected phase and group velocities that have opposite signs. These are the above-mentioned inverse modes.

For any given $K_{y}$, equation (20) has four solutions, only two of which correspond to outgoing waves. Of these two outgoing waves, the one for which the direction of the 
polarization vector, $\boldsymbol{D}$, is closer to the direction of propagation of the wave is called quasi$P$ wave and the one for which $\boldsymbol{D}$ is closer to direction perpendicular to the direction of propagation is called quasi-S wave. However, this distinction is not always valid in materials in which the anisotropy is strong.

In order to find which solutions of (20) are the ones corresponding to outgoing wave, one must examine the direction of the normal to the slowness diagram at the considered point (solution), to make sure that it is indeed an outward-pointing direction. The phase velocity is obtained on this diagram from the direction of the vector connecting the origin with the considered point.

\subsection{Basic scalar absorbing boundary condition for anisotropic elastodynamics}

Assuming the outgoing direction coincides with the positive direction of the $x$ axis, we calculate the group velocity of the solution in the $x$ direction. To this end, we write the solution of (20) for $\omega$ as

$$
\omega= \pm \sqrt{\frac{1}{2 \rho}\left(E_{11}+E_{22} \pm \sqrt{\left(E_{11}-E_{22}\right)^{2}+4 E_{12}^{2}}\right)} .
$$

This can be written more explicitly as $\omega=\omega\left(k_{x}, k_{y}\right)$. Differentiating this expression with respect to $k_{x}$ yields

$$
V_{x}^{g}=\frac{\partial \omega}{\partial k_{x}}=\frac{4 A_{0} k_{x}^{3}+3 A_{1} k_{x}^{2} k_{y}+2 A_{2} k_{x} k_{y}^{2}+A_{3} k_{y}^{3}-\left(2 B_{0} k_{x}+B_{1} k_{y}\right) \rho \omega^{2}}{2\left(B_{0} k_{x}^{2}+B_{1} k_{x} k_{y}+B_{2} k_{y}^{2}\right) \rho \omega-4 \rho^{2} \omega^{3}} .
$$

To distinguish between wavenumbers that give positive and negative outgoing component of the group velocity we need to make simplifying assumptions. The first simplifying approximation we make is that $k_{y}$ is small relative to $k_{x}$, which is equivalent to $s_{y}$ being small relative to $s_{x}$, which is also equivalent to requiring that the basic $\mathrm{ABC}$ we construct is satisfied for small angles of incidence. Thus, $k_{x}$ will satisfy

$$
k_{x}=\alpha-\beta k_{y}+O\left(k_{y}^{2}\right) .
$$

The minus sign before $\beta k_{y}$ is taken for convenience in the subsequent derivation. This results in the approximate dispersion relation

$$
F=A_{0}\left(\alpha^{4}-4 \alpha^{3} \beta k_{y}\right)+A_{1} \alpha^{3} k_{y}-B_{0}\left(\alpha^{2}-2 \alpha \beta k_{y}\right) \rho \omega^{2}-B_{1} \alpha k_{y} \rho \omega^{2}+\rho^{2} \omega^{4}+O\left(k_{y}^{2}\right)=0,
$$

which, after collecting the 0 -th order and 1-st order terms, gives the following equations:

$$
\begin{aligned}
& \left(c_{11} c_{33}-c_{13}^{2}\right) \alpha^{4}-\left(c_{11}+c_{33}\right) \rho \omega^{2} \alpha^{2}+\rho^{2} \omega^{4}=0 \\
& -2\left(c_{11} c_{33}-c_{13}^{2}\right) \alpha^{3} \beta+\left(c_{11} c_{23}-c_{12} c_{23}\right) \alpha^{3}+\left(c_{11}+c_{33}\right) \rho \omega^{2} \alpha \beta-\left(c_{13}+c_{23}\right) \rho \omega^{2} \alpha=0 .
\end{aligned}
$$


Solving, we obtain

$$
\begin{aligned}
& \alpha= \pm \sqrt{\frac{c_{11}+c_{33} \pm \sqrt{\left(c_{11}-c_{33}\right)^{2}+4 c_{13}^{2}}}{2\left(c_{11} c_{33}-c_{13}^{2}\right) /\left(\rho \omega^{2}\right)}}= \pm \omega \sqrt{\frac{\rho}{\chi_{ \pm}\left(c_{i j}\right)}} \\
& \beta=\frac{\left(c_{11} c_{23}-c_{12} c_{13}\right)-\left(c_{13}+c_{23}\right) \chi_{ \pm}}{2\left(c_{11} c_{33}-c_{13}^{2}\right)-\left(c_{11}+c_{33}\right) \chi_{ \pm}}
\end{aligned}
$$

where we define the two constants

$$
\chi_{ \pm}\left(c_{i j}\right)=\frac{2\left(c_{11} c_{33}-c_{13}^{2}\right)}{c_{11}+c_{33} \pm \sqrt{\left(c_{11}-c_{33}\right)^{2}+4 c_{13}^{2}}} .
$$

These constants are positive. To see this, note that the numerator in (37) is positive due to the positive-definiteness of $c_{i j}$. As to the denominator, we use the identity

$$
c_{11}+c_{33} \pm \sqrt{\left(c_{11}-c_{33}\right)^{2}+4 c_{13}^{2}}=c_{11}+c_{33} \pm \sqrt{\left(c_{11}+c_{33}\right)^{2}-4\left(c_{11} c_{33}-c_{13}^{2}\right)} .
$$

Thus, the square root expression is smaller than $c_{11}+c_{33}$, hence the denominator in (37) is also positive. Like $\beta$, the constants $\chi_{ \pm}$depend on the elastic coefficients only. As a result, the first approximation of the relation (31) is linear in $\boldsymbol{k}$ and $\omega$ :

$$
k_{x}= \pm \sqrt{\frac{\rho}{\chi_{ \pm}}} \omega-\beta k_{y}
$$

Thus, dividing (39) by $\omega$, we see that the phase velocity vector in the general anisotropic case will not be a constant, and will in fact be different for different angles of incidence. We shall denote the zero-incidence-angle phase velocities of the quasi-P and quasi-S waves for convenience purposes as follows:

$$
c_{L}=\sqrt{\frac{\chi_{+}}{\rho}} ; \quad c_{T}=\sqrt{\frac{\chi_{-}}{\rho}} .
$$

By transforming the result (39) back into physical space $\left(k_{x} \rightarrow i \partial_{x}, k_{y} \rightarrow i \partial_{y}, \omega \rightarrow-i \partial_{t}\right.$, where $\partial_{i}$ denotes partial differentiation with respect to the variable $i$ ), we obtain four scalar conditions for the anisotropic case, i.e.,

$$
\begin{aligned}
& \left(\partial_{t} \pm c_{L} \partial_{x} \pm c_{L} \beta \partial_{y}\right) \boldsymbol{u}=\mathbf{0} \\
& \left(\partial_{t} \pm c_{T} \partial_{x} \pm c_{T} \beta \partial_{y}\right) \boldsymbol{u}=\mathbf{0}
\end{aligned}
$$

where the same sign must be taken before the two spatial derivatives in each of (41) and (42). A particular selection of the two plus or minus signs in (41) and (42) corresponds to making an assumption of the outgoing direction. However, any such selection cannot guarantee that all waves that propagate in the medium at this boundary will indeed be outgoing, since this simple $\mathrm{ABC}$ does not take inverse modes into account in its derivation, and cannot be expected to perform well when such modes are present. Indeed, we show below for the 
particular case of an orthotropic material that this condition assumes the wrong direction of the group velocity when such modes occur.

In (41) and (42), two variants of the basic ABC are obtained, one of which, (41), absorbs quasi-P waves, and the other, (42), absorbs quasi-S waves, which are valid for small angles of incidence, for incident waves that contain no inverse modes. The above conditions (41) and (42) can be merged together into a single boundary condition of the form

$$
\left(a \partial_{t}+c_{L} \partial_{x}+c_{L} \beta \partial_{y}\right) \boldsymbol{u}=\mathbf{0}
$$

where $a$ is a numeric parameter to be chosen, with the choice being equivalent to choosing any velocity in the spatial derivative terms, instead of $c_{L}$, as done in a similar manner in the the Higdon ABC for isotropic media [38]. The constants a can be optimized in order to obtain the best performance of the boundary condition. The constant $\beta$ is a measure of the amount of anisotropy of the material, as shown in (36).

If we now make a second simplifying assumption, that the elastic material is orthotropic, i.e., $c_{13}=c_{23}=0$, this gives $\chi_{+}=c_{11}, \chi_{-}=c_{33}$, and the following simple expressions are obtained for the four values of $\alpha$ that solve (33), and the corresponding value of $\beta$ :

$$
\alpha_{1,2}= \pm \sqrt{\frac{\rho \omega^{2}}{c_{11}}}, \quad \alpha_{3,4}= \pm \sqrt{\frac{\rho \omega^{2}}{c_{33}}}, \quad \beta_{1,2,3,4}=0 .
$$

When these values of $\alpha$ are substituted into (31) we obtain

$$
V_{x}^{p}=\frac{\omega}{k_{x}}= \pm \sqrt{\frac{c_{11}}{\rho}}, \quad \text { or } \quad V_{x}^{p}= \pm \sqrt{\frac{c_{33}}{\rho}} .
$$

The resulting phase velocities are the velocities of the quasi-P and quasi-Q waves, respectively. The assumption of small incidence angle in the orthotropic case therefore translates into a phase velocity that does not depend on the incidence angle.

The group velocity in the $x$ direction, under the two assumptions made (small incidence angles and orthotropy), reduces to

$$
V_{x}^{g} \approx \frac{k_{x}}{\omega} \frac{2 \frac{c_{11} c_{33}}{\rho^{2}} \frac{k_{x}^{2}}{\omega^{2}}-\frac{\left(c_{11}+c_{33}\right)}{\rho}}{\frac{\left(c_{11}+c_{33}\right)}{\rho} \frac{k_{x}^{2}}{\omega^{2}}-2} .
$$

We note that (46) can be obtained from (30) by neglecting the $O\left(k_{y}^{2}\right)$ terms and taking into account that $A_{1}=B_{1}=0$ for orthotropic materials. When each of the phase velocities (45) are substituted into (46), the corresponding obtained group velocities are

$$
V_{x}^{g}=\operatorname{sgn}\left(V_{x}^{p}\right) \sqrt{\frac{c_{11}}{\rho}}, \quad \operatorname{sgn}\left(V_{x}^{p}\right) \sqrt{\frac{c_{33}}{\rho}} .
$$

Here "sgn" stands for the sign function. Thus at this approximation the group velocity of all waves has the same sign as the phase velocity, and the basic ABC put forth by this 
approximation does not take into account the fact that outgoing waves may exist in the medium for which the sign of the phase velocity is opposite to the sign of the group velocity, as one can see for example in [27].

A basic ABC for an orthotropic material on a right boundary is obtained from (31) by substituting either of the positive values of $k_{x}$ from (44) together with $\beta=0$, neglecting the $O\left(k_{y}^{2}\right)$ terms and transforming the result back into physical space. The same result can also be obtained by setting $c_{13}=c_{23}=0$ in (41):

$$
\begin{array}{rlrl}
\left(\partial_{t}+c_{L} \partial_{x}\right) \boldsymbol{u} & =\mathbf{0}, & \text { or } \\
\left(\partial_{t}+c_{T} \partial_{x}\right) \boldsymbol{u} & =\mathbf{0} .
\end{array}
$$

Here we have $c_{L}=\sqrt{c_{11} / \rho}, c_{T}=\sqrt{c_{33} / \rho}$.

The practical boundary condition equivalent to (43) is

$$
\left(a \partial_{t}+c_{L} \partial_{x}\right) \boldsymbol{u}=\mathbf{0} .
$$

These basic ABCs, both in the orthotropic and in general anisotropic case, are scalar conditions, which as we shall see, have certain advantages in the construction of the DAB scheme based on such conditions.

We note that the construction of conditions based on near-normal incidence is typically not effective for long time computations. For isotropic problems we have shown that the use of parameters optimized for acoustics can be more effective over long times [14,20]. These involve the addition of zero-order terms to (43). The problem of directly optimizing parameters in the elastic case is still under study.

\subsection{Vectorial absorbing boundary condition for anisotropic elastodynamics}

In addition to the scalar $\mathrm{ABC}$ developed above, the $\mathrm{DAB}$ scheme requires a vectorial $\mathrm{ABC}$ as a final stage in the sequence that together forms the high-order condition. We use here energy decay considerations to develop such a vectorial condition, namely this condition should assure that the rate of change of energy density in time is always negative.

The total energy density is the sum of potential and kinetic energies, i.e.,

$$
E(t)=\frac{1}{2} \int_{\Omega}\left(\rho u_{i, t} u_{i, t}+C_{i j k l} e_{i j} e_{k l}\right) d \Omega=\frac{1}{2} \int_{\Omega}\left(\rho u_{i, t} u_{i, t}+C_{i j k l} u_{i, j} u_{k, l}\right) d \Omega
$$

where $e_{i j}=\frac{1}{2}\left(u_{i, j}+u_{j, i}\right)$ are the strain tensor components, and we make use of the symmetric properties of $C_{i j k l}$, namely $C_{i j k l}=C_{j i k l}=C_{i j l k}$. Here, $\Omega$ is the two-dimensional domain of the problem.

Taking the time derivative of the energy, we have

$$
\frac{d E}{d t}=\int_{\Omega}\left(\rho u_{i, t} u_{i, t t}+\frac{C_{i j k l}}{2}\left(u_{i, j t} u_{k, l}+u_{i, j} u_{k, l t}\right)\right) d \Omega .
$$


Using (1) and (2), and assuming $f_{i}=0$, we obtain

$$
\frac{d E}{d t}=\frac{1}{2} \int_{\Omega} C_{i j k l}\left(u_{i, t} u_{k, l j}+u_{i, t} u_{l, k j}+u_{i, j t} u_{k, l}+u_{i, j} u_{k, l t}\right) d \Omega
$$

Integrating by parts the first two terms of the integrand with respect to the variable $j$, and using the symmetry $C_{i j k l}=C_{k l i j}$, the integral over $\Omega$ cancels out, and we obtain that the energy changes on the boundary $\Gamma$ only, i.e.,

$$
\frac{d E}{d t}=\frac{1}{2} \int_{\Gamma} C_{i j k l}\left(u_{i, t} u_{k, l} n_{j}+u_{i, t} u_{l, k} n_{j}\right) d \Gamma
$$

where $\boldsymbol{n}=\left\{n_{j}\right\}$ is the outward unit vector that is perpendicular to the boundary. We consider here the rate of energy change on the Eastern boundary $\Gamma_{E}$, in other words $j=1$. Written out fully (54) becomes

$$
\begin{aligned}
\frac{d E}{d t}= & \int_{\Gamma_{E}} C_{1111} u_{x, t} u_{x, x} d \Gamma+\int_{\Gamma_{E}} C_{1122} u_{x, t} u_{y, y} d \Gamma \\
& +\frac{1}{2} \int_{\Gamma_{E}} C_{1112} u_{x, t}\left(u_{x, y}+u_{y, x}\right) d \Gamma+\frac{1}{2} \int_{\Gamma_{E}} C_{1121} u_{x, t}\left(u_{y, x}+u_{x, y}\right) d \Gamma \\
& +\int_{\Gamma_{E}} C_{2111} u_{y, t} u_{x, x} d \Gamma+\int_{\Gamma_{E}} C_{2122} u_{y, t} u_{y, y} d \Gamma \\
& +\frac{1}{2} \int_{\Gamma_{E}} C_{2112} u_{y, t}\left(u_{x, y}+u_{y, x}\right) d \Gamma+\frac{1}{2} \int_{\Gamma_{E}} C_{2121} u_{y, t}\left(u_{y, x}+u_{x, y}\right) d \Gamma \\
= & \int_{\Gamma_{E}} c_{11} u_{x, t} u_{x, x} d \Gamma+\int_{\Gamma_{E}} c_{12} u_{x, t} u_{y, y} d \Gamma+\int_{\Gamma_{E}} c_{13} u_{x, t}\left(u_{x, y}+u_{y, x}\right) d \Gamma \\
& +\int_{\Gamma_{E}} c_{31} u_{y, t} u_{x, x} d \Gamma+\int_{\Gamma_{E}} c_{32} u_{y, t} u_{y, y} d \Gamma+\int_{\Gamma_{E}} c_{33} u_{y, t}\left(u_{x, y}+u_{y, x}\right) d \Gamma \\
= & \int_{\Gamma_{E}} \boldsymbol{u}_{, t}^{T}\left(\boldsymbol{c}^{* *} \boldsymbol{u}_{, x}+\boldsymbol{c}^{*} \boldsymbol{u}_{, y}\right) d \Gamma,
\end{aligned}
$$

where we have defined the following matrices:

$$
\boldsymbol{c}^{*}=\left[\begin{array}{ll}
c_{13} & c_{12} \\
c_{33} & c_{32}
\end{array}\right] ; \quad \boldsymbol{c}^{* *}=\left[\begin{array}{ll}
c_{11} & c_{13} \\
c_{31} & c_{33}
\end{array}\right] .
$$

Thus, if we apply on $\Gamma_{E}$ the boundary condition

$$
\boldsymbol{c}^{* *} \boldsymbol{u}_{, x}+\boldsymbol{c}^{*} \boldsymbol{u}_{, y}+\boldsymbol{s} \boldsymbol{u}_{, t}=\boldsymbol{T}+\boldsymbol{s} \boldsymbol{u}_{, t}=\mathbf{0}
$$

where $\boldsymbol{T}$ is the traction vector on $\Gamma_{E}$ and $s$ is a positive semidefinite matrix, then we will have

$$
\frac{d E}{d t}=-\int_{\Gamma_{E}} \boldsymbol{u}_{, t}^{T} \boldsymbol{s} \boldsymbol{u}_{, t} \leq 0 .
$$

which means that since the energy always decreases in time, this condition is always stable. Usually we take $s$ in the LK-like form (see [21]),

$$
\boldsymbol{s}=\boldsymbol{s}^{L K}=\left[\begin{array}{cc}
c_{L} & 0 \\
0 & c_{T}
\end{array}\right]
$$




\section{The Double Absorbing Boundary (DAB) Method for Problems in Anisotropic Elastodynamics}

\subsection{The problem in the computational domain}

We now truncate the semi-infinite domain by introducing the artificial boundary $\Gamma_{E}$, located at $x=x_{E}$ and spanning $0 \leqslant y \leqslant b$. Slightly to the west of $\Gamma_{E}$ we set an interface denoted $\Gamma_{I}$, located at $x=x_{I}$, with $0 \leqslant y \leqslant b$. See Fig. 1(b). The entire computational domain bounded by $\Gamma_{N} \cup \Gamma_{W} \cup \Gamma_{S} \cup \Gamma_{E}$ is denoted by $\Omega$. As Fig. 1(b) shows, this domain is divided by the interface $\Gamma_{I}$ into two sub-domains: the interior domain $\Omega_{I}$ and a thin layer $\Omega_{L}$. We choose the location of $\Gamma_{E}$ and $\Gamma_{I}$ such that $\Omega_{0}$ is strictly contained within $\Omega_{I}$. Thus, in the layer $\Omega_{L}$ we have that $C_{i j k l}$ is constant, $f_{i}=0$, and the initial conditions are zero.

The function $\boldsymbol{u}$ satisfies the equation of elastodynamics (1) in $\Omega$, the periodic lateral boundary conditions (7), the west boundary conditions (11), (12), and the initial conditions (13) and (14) in $\Omega$. In the layer $\Omega_{L}$ we shall apply a special treatment, with the goal of rendering the solution in the interior domain $\Omega_{I}$ as close as possible to the solution of the original semi-infinite problem in that domain. Thus, $\Omega_{L}$ will act as an absorbing or nonreflecting layer.

To this end we define a sequence of auxiliary variables $\phi_{i}^{0}, \ldots, \phi_{i}^{P}, i=x, y$ in the layer only. Here $P$ is a chosen parameter that will determine the order of accuracy of the absorbing layer. The first auxiliary variable is defined to be $\phi_{i}^{0}=u_{i}$ in $\Omega_{L}$. The problem for the $\phi_{i}^{m}$ is given as follows. In the layer, we require the $\phi_{i}^{m}$ for a particular value of $m$ to satisfy the same elastic wave equation as for $u_{i}$, i.e. (1), with $f_{i}=0$ :

$$
\rho \ddot{\phi}_{i}^{m}=\sigma_{i j, j}\left(\phi_{i}^{m}\right), \quad m=1, \ldots, P .
$$

We also denote by $T_{i}^{m}$ the traction vector on $\Gamma_{I}$ and $\Gamma_{E}$ that corresponds to the variables $\phi_{i}^{m}$. Also, $T_{i}=T_{i}^{0}$.

All the auxiliary variables satisfy a zero initial state:

$$
\phi_{i}^{m}(x, y, t=0)=0, \quad \dot{\phi}_{i}^{m}(x, y, t=0)=0, \quad m=1, \ldots, P, \quad \text { in } \quad \Omega_{L} .
$$

On $\Gamma_{S}$ and $\Gamma_{N}$ (or more precisely the parts of these boundaries that are in the layer, i.e., for $x_{I} \leq x \leq x_{E}$ and $0 \leq y \leq b$ ) we apply the same (periodic) boundary conditions as for $u_{i}$, i.e.,

$$
\begin{aligned}
& \phi_{i}^{m}(x, 0, t)=\phi_{i}^{m}(x, b, t) \\
& \phi_{i, y}^{m}(x, 0, t)=\phi_{i, y}^{m}(x, b, t), \quad m=1, \ldots, P, \quad \text { on } \quad \Gamma_{S}, \Gamma_{N} .
\end{aligned}
$$

Now we need to define boundary conditions for the $\phi_{i}^{m}$ on $\Gamma_{I}$ and on $\Gamma_{E}$. The boundary conditions on these boundaries will combine the basic scalar ABC, that will be defined for each different $m$, in a recursive manner, as well as the vectorial $\mathrm{ABC}$, which will terminate 
the recursion. This setup is needed in order for the reflection coefficient to decrease with the order of $P$ (see [39], [15], [14]).

The recursive (scalar) boundary conditions are defined in a manner which is similar to the ABC for isotropic elasticity defined in [39]. Each relation has the form $B^{+} \phi_{i}^{m}=B^{-} \phi_{i}^{m+1}$, where $B^{+}$is an operator that admits only outgoing waves, and $B^{-}$is an operator that admits only incoming waves. It can be shown, as in [40], that this form has several advantages over the simpler form $B^{+} \phi_{i}^{m}=\phi_{i}^{m+1}$ proposed in [41], one of which is that the former has a twice as high rate of convergence. In the present case, the $B^{+}$operator is the basic $\mathrm{ABC}$ operator defined in (43), while the $B^{-}$operator is the corresponding operator for incoming waves, with appropriately changed signs. Precisely, the recursive relations are

$$
\begin{gathered}
a_{m} \dot{\phi}_{x}^{m}+c_{L} \phi_{x, x}^{m}+\beta c_{L} \phi_{x, y}^{m}=a_{m} \dot{\phi}_{x}^{m+1}-c_{L} \phi_{x, x}^{m+1}-\beta c_{L} \phi_{x, y}^{m+1} \\
a_{m} \dot{\phi}_{y}^{m}+c_{L} \phi_{y, x}^{m}+\beta c_{L} \phi_{y, y}^{m}=a_{m} \dot{\phi}_{y}^{m+1}-c_{L} \phi_{y, x}^{m+1}-\beta c_{L} \phi_{y, y}^{m+1} \\
\text { with } \quad m=0, \ldots, P-1, \quad \text { on } \quad \Gamma_{I}, \Gamma_{E} .
\end{gathered}
$$

In (64) and (65), $a_{m}$ are the parameters of the boundary conditions as defined in the "Computational Aspects" section below. Note also that in the orthotropic case, one simply has to substitute $\beta=0$ and use $c_{L}=\sqrt{c_{11} / \rho}$. To complete the recursive definition, we require the following vectorial termination condition on $\Gamma_{E}$, as developed above:

$$
\begin{aligned}
& T_{x}^{P}+\rho c_{L} \dot{\phi}_{x}^{P}=0 \\
& T_{y}^{P}+\rho c_{T} \dot{\phi}_{y}^{P}=0 .
\end{aligned}
$$

In these equations, the traction variables $T_{i}^{P}$ are related to the displacement variables $\phi_{i}^{P}$ via

$$
T_{i}^{P}=c_{i j k l} n_{j} \phi_{k, l}^{P}
$$

similarly to (10). In this manner, using the recursion, the scalar boundary condition is extended to high orders, and is terminated using the LK-like condition.

Fig. 3 illustrates the "ladder" structure of the DAB scheme, namely the flow of information on the two boundaries bounding the DAB layer. Conceptually, the information flows in different directions on the two boundaries. On $\Gamma_{I}$, the variable $u_{i}=\phi_{i}^{0}$ can be calculated from the interior scheme, and then $\phi_{i}^{1}$ can be calculated from the first recursive relation. Then $\phi_{i}^{2}$ can be calculated from the second recursive relation, and so on. Thus, on $\Gamma_{I}$ the natural flow of information corresponds to $m=0,1, \ldots, P$. On the other hand, on $\Gamma_{E}$, the termination conditions (66) and (67) are imposed as boundary conditions for $\phi_{i}^{P}$, and then $\phi_{i}^{P-1}$ can be found from the last recursion relation, and so on, in a backward progress. Thus, on $\Gamma_{E}$ the natural flow of information corresponds to $m=P, P-1, \ldots, 0$. An explicit implementation of this description is actually taken in [13] using a finite difference scheme. In the present work, however, we solve the interior equations and all the boundary equations as one big system. 


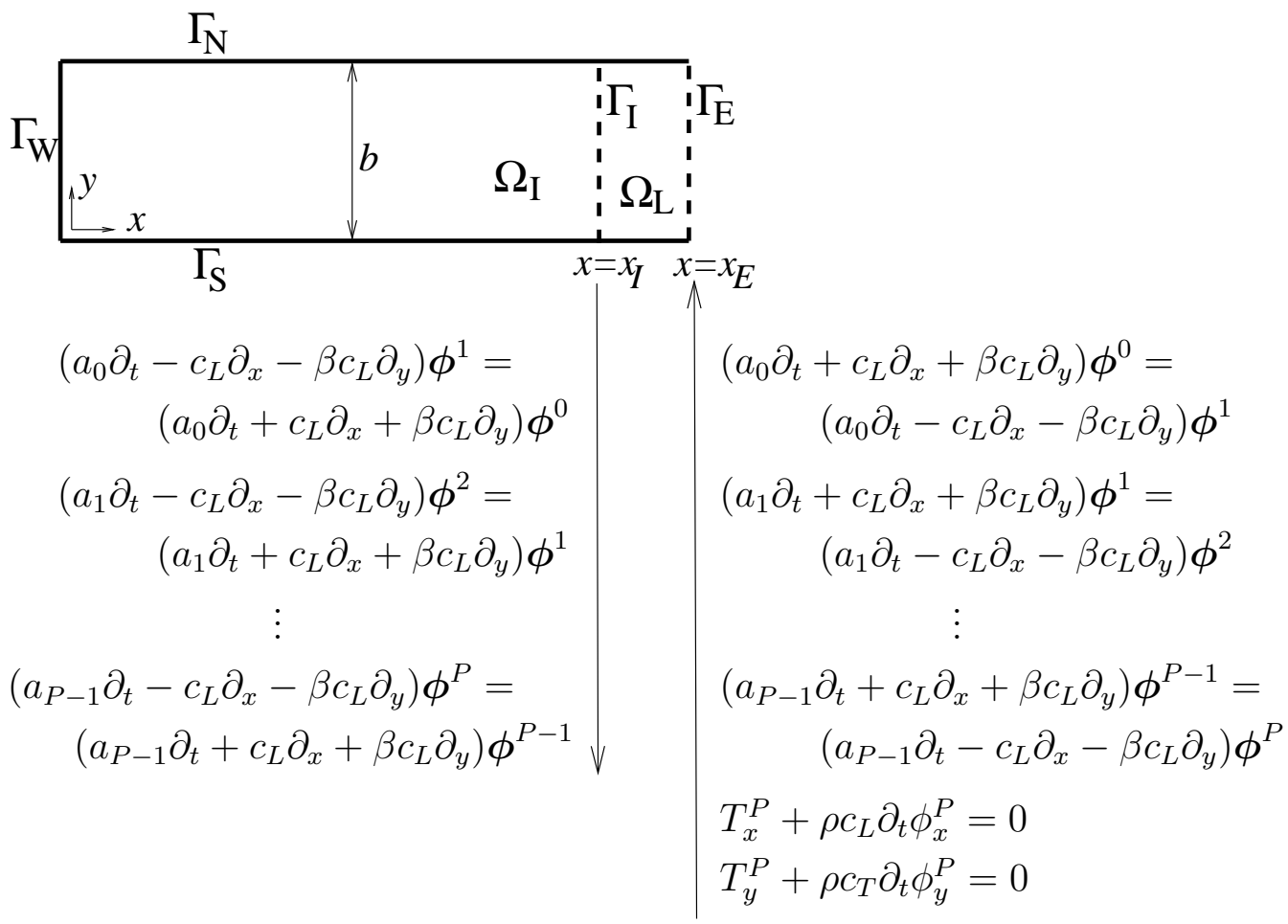

Figure 3: The "ladder" structure of the DAB, showing the flow of information on the two boundaries bounding the layer.

\subsection{The DAB conditions for a layered medium}

In the previous subsections we considered a homogeneous anisotropic medium. Now we consider the case of a layered medium, with different material properties in the different layers. The case of isotropic layers was considered in [15], and here we allow the layers to be anisotropic. The layers are assumed to be perpendicular to the absorbing boundary, namely the longitudinal direction inside the layer is the $x$ direction. The jump conditions along the interfaces between layers are

$$
\begin{aligned}
& \llbracket \phi_{x}^{m} \rrbracket=0 ; \quad \llbracket \phi_{y}^{m} \rrbracket=0 \\
& \llbracket T_{x}^{m} \rrbracket=\llbracket \sigma_{y x}^{m} \rrbracket=0 ; \quad \llbracket T_{y}^{m} \rrbracket=\llbracket \sigma_{y y}^{m} \rrbracket=0 .
\end{aligned}
$$

Here $\llbracket \cdot \rrbracket$ denoted a jump across the interface between two layers. In the above conditions, the $\sigma_{i j}^{m}$ are related to the $\phi_{i}^{m}$ via

$$
\sigma_{i j}^{m}=c_{i j k l} \phi_{k, l}^{m}
$$

By dividing the expression for the recursive relations (64) and (65) by $a_{m}^{(n)}$, where the 
superscript $(n)$ indicates the $n$-th layer, we obtain for each layer $n$ :

$$
\begin{array}{r}
\dot{\phi}_{i}^{m}+\frac{c_{L}^{(n)}}{a_{m}^{(n)}} \phi_{i, x}^{m}+\frac{\beta c_{L}^{(n)}}{a_{m}^{(n)}} \phi_{i, y}^{m}=\dot{\phi}_{i}^{m+1}-\frac{c_{L}^{(n)}}{a_{m}^{(n)}} \phi_{i, x}^{m+1}-\frac{\beta c_{L}^{(n)}}{a_{m}^{(n)}} \phi_{i, y}^{m+1} \\
\text { with } \quad m=0, \ldots, P-1 ; \quad i=x, y, \quad \text { on } \quad \Gamma_{I}, \Gamma_{E} .
\end{array}
$$

Since the auxiliary variables $\phi_{i}^{m}$ and their derivatives in the $x$ direction and in time are continuous between the layers, there are two conclusions:

1. We will require that the ratio $\frac{c_{L}^{(n)}}{a_{m}^{(n)}}$ also be continuous between the layers, namely that it does not depend on $n$.

2. Since the derivatives of the variables $\phi_{i}^{m}$ in the $y$ direction are not continuous, (72) cannot hold in the case of a general anisotropic material. We shall therefore focus on the orthotropic case, in which $\beta=0$.

Thus, in the orthotropic case, if we denote by an asterisk some reference value of the corresponding parameter, by requiring that

$$
\frac{c_{L}^{(n)}}{a_{m}^{(n)}}=\frac{c_{L}^{*}}{a_{m}^{*}},
$$

we obtain that all the recursive relations (72) for the different layers can be replaced by the following recursive relation written out for a reference layer:

$$
\begin{aligned}
\dot{\phi}_{i}^{m}+\frac{c_{L}^{*}}{a_{m}^{*}} \phi_{i, x}^{m} & =\dot{\phi}_{i}^{m+1}-\frac{c_{L}^{*}}{a_{m}^{*}} \phi_{i, x}^{m+1} \\
\text { with } \quad m & =0, \ldots, P-1 ; \quad i=x, y, \quad \text { on } \quad \Gamma_{I}, \Gamma_{E} .
\end{aligned}
$$

Now we consider the termination conditions for the different layers, and their continuity across the layer interfaces. Let $\gamma$ denote an interface between two layers, and let the intersection point of $\gamma$ and $\Gamma_{E}$ be denoted by $\eta$. Then, at the point $\eta$ we have

$$
T_{y}^{m}=\sigma_{x y}^{m}=\sigma_{y x}^{m}=T_{x}^{m}
$$

Therefore, at the intersection of $\Gamma_{E}$ and the interface between the layers, we obtain from the second termination condition (67) that

$$
T_{x}^{P}+\rho c_{T}^{(n)} \dot{\phi}_{y}^{P}=0
$$

which cannot hold since $T_{x}^{P}, \dot{\phi}_{y}^{P}$ are continuous, while $\rho c_{T}^{(n)}$ is not. However, in practice, any positive wave speed can be substituted for $c_{T}$ in (67) without losing stability. The reduced accuracy will be compensated for by the recursive relations. 
Therefore, in the case of layered media we take the following termination conditions:

$$
\begin{aligned}
& T_{x}^{P}+\rho c_{L}^{*} \dot{\phi}_{x}^{P}=0 \\
& T_{y}^{P}+\rho c_{T}^{*} \dot{\phi}_{y}^{P}=0 .
\end{aligned}
$$

Thus, together (74), (77) and (78) are the modified DAB equations for the case of layered media. The spatial discretization in this case is carried out in the same manner as for the homogeneous medium using the starred parameters $a_{m}^{*}, \tilde{a}_{m}^{*}, b_{m}^{*}, \tilde{b}_{m}^{*}, c_{L}^{*}, c_{T}^{*}$ which hold in a chosen reference layer.

\section{Finite Element Formulation}

The FE formulation developed in this section holds for any anisotropic material. In our numerical examples we shall specialize it to orthotropic materials.

\subsection{Variational formulation}

The strong form of the DAB scheme consists of the elastic equations (1) in $\Omega$ and (60) in $\Omega_{L}$, the initial conditions (13), (14) in $\Omega$ and (61) in $\Omega_{L}$, the periodical boundary conditions (7), (8) and (62), (63) on $\Gamma_{S}$ and $\Gamma_{N}$, the boundary conditions (11) and (12), and the recursive boundary relations (64) and (65) on $\Gamma_{I}$ and $\Gamma_{E}$, with the termination conditions (66) and (67) on $\Gamma_{E}$.

To state the weak form we first define the following function spaces:

$$
\begin{aligned}
& \mathcal{S}=\left\{\boldsymbol{u} \equiv\left\{u_{i}\right\} \mid\left\{u_{i}\right\} \in H^{1}(\Omega), u_{i}\left(\Gamma_{S}\right)=u_{i}\left(\Gamma_{N}\right), u_{i}\left(\Gamma_{W}\right)=0\right\} \\
& \mathcal{S}_{L}=\left\{\boldsymbol{\phi}^{m} \equiv\left\{\phi_{i}^{m}\right\} \mid\left\{\phi_{i}^{m}\right\} \in H^{1}\left(\Omega_{L}\right), \phi_{i}^{m}\left(\Gamma_{S}\right)=\phi_{i}^{m}\left(\Gamma_{N}\right), m=1, \ldots P\right\}
\end{aligned}
$$

Note that the periodicity of the functions $u_{i}$ and $\phi_{i}^{m}$ (but not of their $y$ derivatives) is enforced strongly, by being built into the trial and test spaces.

We multiply equation (1) by a weight function $\boldsymbol{w}^{0} \in \mathcal{S}$ and the equations (60) by the weight functions $\boldsymbol{w}^{m} \in \mathcal{S}_{L}, m=1, \ldots, P$, and integrate the terms that include $C_{i j k l}$ by parts. This yields the weak equations that will be written below. A term included in the weak equation for $\boldsymbol{u}$ is the integral on the lateral boundaries

$$
B_{N S}^{0}=\int_{\Gamma_{N} \cup \Gamma_{S}} w_{i}^{0} T_{i}^{0} d \Gamma
$$

However, owing to (9), the traction $\boldsymbol{T}^{0}$ has an opposite sign on $\Gamma_{N}$ and $\Gamma_{S}$, whereas $\boldsymbol{w}^{0}$ is the same on the two boundaries due to the strong enforcement of the periodicity in (79). This implies $B_{N S}^{0}=0$. Note that whereas the periodicity of the function $\boldsymbol{u}$ is enforced strongly, the periodicity of $\boldsymbol{u}_{, y}$ is enforced weakly. A similar situation exists for any of the auxiliary variables $\phi^{m}$. 
The weak equation for $\boldsymbol{u}$ is

$$
\begin{aligned}
& \int_{\Omega} w_{i}^{0} \rho u_{i, t t} d \Omega+\int_{\Omega} w_{i, j}^{0} C_{i j k l} u_{k, l} d \Omega+B_{E}^{0}=\int_{\Omega} w_{i}^{0} f_{i} d \Omega \\
& \int_{\Omega_{L}} w_{i}^{m} \rho \phi_{i, t t}^{m} d \Omega+\int_{\Omega_{L}} w_{i, j}^{m} C_{i j k l} \phi_{k, l}^{m} d \Omega+B_{I}^{m}+B_{E}^{m}=0, \quad m=1, \ldots, P,
\end{aligned}
$$

where we have

$$
\begin{aligned}
& B_{I}^{m}=-\int_{\Gamma_{I}}\left[w_{x}^{m} T_{x}^{m}+w_{y}^{m} T_{y}^{m}\right] d \Gamma \\
& B_{E}^{m}=-\int_{\Gamma_{E}}\left[w_{x}^{m} T_{x}^{m}+w_{y}^{m} T_{y}^{m}\right] d \Gamma .
\end{aligned}
$$

Note that the definition (84) applies to $m=1, \ldots, P$ whereas the definition (85) applies to $m=0, \ldots, P$.

We rewrite the recursive relations (64) and (65) in the following form. On the boundary $\Gamma_{I}$ :

$$
\begin{aligned}
\phi_{x, x}^{m} & =\frac{a_{m-1}}{c_{L}}\left(\dot{\phi}_{x}^{m}-\dot{\phi}_{x}^{m-1}\right)-\beta\left(\phi_{x, y}^{m}+\phi_{x, y}^{m-1}\right)-\phi_{x, x}^{m-1} \\
\phi_{y, x}^{m} & =\frac{a_{m-1}}{c_{L}}\left(\dot{\phi}_{y}^{m}-\dot{\phi}_{y}^{m-1}\right)-\beta\left(\phi_{y, y}^{m}+\phi_{y, y}^{m-1}\right)-\phi_{y, x}^{m-1} \\
m & =1, \ldots, P, \quad \text { on } \quad \Gamma_{I}
\end{aligned}
$$

On the boundary $\Gamma_{E}$ :

$$
\begin{aligned}
\phi_{x, x}^{m} & =\frac{a_{m}}{c_{L}}\left(\dot{\phi}_{x}^{m+1}-\dot{\phi}_{x}^{m}\right)-\beta\left(\phi_{x, y}^{m+1}+\phi_{x, y}^{m}\right)-\phi_{x, x}^{m+1} \\
\phi_{y, x}^{m} & =\frac{a_{m}}{c_{L}}\left(\dot{\phi}_{y}^{m+1}-\dot{\phi}_{y}^{m}\right)-\beta\left(\phi_{y, y}^{m+1}+\phi_{y, y}^{m}\right)-\phi_{y, x}^{m+1} \\
m & =0, \ldots, P-1, \quad \text { on } \quad \Gamma_{E} .
\end{aligned}
$$

As the termination conditions we use (66) and (67), i.e.,

$$
\begin{aligned}
& T_{x}^{P}=-\rho c_{L} \dot{\phi}_{x}^{P} \\
& T_{y}^{P}=-\rho c_{T} \dot{\phi}_{y}^{P}, \quad \text { on } \quad \Gamma_{E},
\end{aligned}
$$

By substituting (86)-(89) in the terms $B_{E}^{m}, B_{I}^{m}$ in (84) and (85), these terms become

$$
\begin{aligned}
B_{I}^{m}= & \int_{\Gamma_{I}} w_{x}^{m}\left[c_{11} \phi_{x, x}^{m}+c_{12} \phi_{y, y}^{m}+c_{13} \phi_{x, y}^{m}+c_{13} \phi_{y, x}^{m}\right] d \Gamma \\
+ & \int_{\Gamma_{I}} w_{y}^{m}\left[c_{31} \phi_{x, x}^{m}+c_{32} \phi_{y, y}^{m}+c_{33} \phi_{x, y}^{m}+c_{33} \phi_{y, x}^{m}\right] d \Gamma= \\
& \int_{\Gamma_{I}} w_{x}^{m}\left[\frac{a_{m-1} c_{11}}{c_{L}} \dot{\phi}_{x}^{m}-\frac{a_{m-1} c_{11}}{c_{L}} \dot{\phi}_{x}^{m-1}-c_{11} \beta \phi_{x, y}^{m}-c_{11} \beta \phi_{x, y}^{m-1}-c_{11} \phi_{x, x}^{m-1}+c_{12} \phi_{y, y}^{m}\right.
\end{aligned}
$$




$$
\begin{gathered}
\left.\quad+\frac{a_{m-1} c_{13}}{c_{L}} \dot{\phi}_{y}^{m}-\frac{a_{m-1} c_{13}}{c_{L}} \dot{\phi}_{y}^{m-1}-c_{13} \beta \phi_{y, y}^{m}-c_{13} \beta \phi_{y, y}^{m-1}-c_{13} \phi_{y, x}^{m-1}+c_{13} \phi_{x, y}^{m}\right] d \Gamma \\
+\int_{\Gamma_{I}} w_{y}^{m}\left[\frac{a_{m-1} c_{31}}{c_{L}} \dot{\phi}_{x}^{m}-\frac{a_{m-1} c_{31}}{c_{L}} \dot{\phi}_{x}^{m-1}-c_{31} \beta \phi_{x, y}^{m}-c_{31} \beta \phi_{x, y}^{m-1}-c_{31} \phi_{x, x}^{m-1}+c_{32} \phi_{y, y}^{m}\right. \\
\left.+\frac{a_{m-1} c_{33}}{c_{L}} \dot{\phi}_{y}^{m}-\frac{a_{m-1} c_{33}}{c_{L}} \dot{\phi}_{y}^{m-1}-c_{33} \beta \phi_{y, y}^{m}-c_{33} \beta \phi_{y, y}^{m-1}-c_{33} \phi_{y, x}^{m-1}+c_{33} \phi_{x, y}^{m}\right] d \Gamma \\
m=1, \ldots, P \text { on } \Gamma_{I} \\
B_{E}^{m}=-\int_{\Gamma_{E}} w_{x}^{m}\left[c_{11} \phi_{x, x}^{m}+c_{12} \phi_{y, y}^{m}+c_{13} \phi_{x, y}^{m}+c_{13} \phi_{y, x}^{m}\right] d \Gamma \\
-\int_{\Gamma_{E}} w_{y}^{m}\left[c_{31} \phi_{x, x}^{m}+c_{32} \phi_{y, y}^{m}+c_{33} \phi_{x, y}^{m}+c_{33} \phi_{y, x}^{m}\right] d \Gamma= \\
-\int_{\Gamma_{E}} w_{x}^{m}\left[\frac{a_{m} c_{11}}{c_{L}} \dot{\phi}_{x}^{m+1}-\frac{a_{m} c_{11}}{c_{L}} \dot{\phi}_{x}^{m}-c_{11} \beta \phi_{x, y}^{m+1}-c_{11} \beta \phi_{x, y}^{m}-c_{11} \phi_{x, x}^{m+1}+c_{12} \phi_{y, y}^{m}\right. \\
\left.\quad+\frac{a_{m} c_{13}}{c_{L}} \dot{\phi}_{y}^{m+1}-\frac{a_{m} c_{13}}{c_{L}} \dot{\phi}_{y}^{m}-c_{13} \beta \phi_{y, y}^{m+1}-c_{13} \beta \phi_{y, y}^{m}-c_{13} \phi_{y, x}^{m+1}+c_{13} \phi_{x, y}^{m}\right] d \Gamma \\
-\int_{\Gamma_{E}} w_{y}^{m}\left[\frac{a_{m} c_{31} \dot{\phi}_{x}^{m+1}-\frac{a_{m} c_{31}}{c_{L}} \dot{\phi}_{x}^{m}-c_{31} \beta \phi_{x, y}^{m+1}-c_{31} \beta \phi_{x, y}^{m}-c_{31} \phi_{x, x}^{m+1}+c_{32} \phi_{y, y}^{m}}{\left.+\frac{a_{m} c_{33}}{c_{L}} \dot{\phi}_{y}^{m+1}-\frac{a_{m} c_{33}}{c_{L}} \dot{\phi}_{y}^{m}-c_{33} \beta \phi_{y, y}^{m+1}-c_{33} \beta \phi_{y, y}^{m}-c_{33} \phi_{y, x}^{m+1}+c_{33} \phi_{x, y}^{m}\right] d \Gamma}\right. \\
\Gamma_{E}=0, \ldots, P-1 \\
\Gamma_{E}
\end{gathered}
$$

The term corresponding to $m=P$ is obtained by substituting (90) and (91) directly into (85):

$$
B_{E}^{P}=\int_{\Gamma_{E}} w_{x}^{P} \rho c_{L} \dot{\phi}_{x}^{P} d \Gamma+\int_{\Gamma_{E}} w_{y}^{P} \rho c_{T} \dot{\phi}_{y}^{P} d \Gamma \quad \text { on } \quad \Gamma_{E}
$$

Here and elsewhere, the $x$-derivatives on the boundaries $\Gamma_{I}, \Gamma_{E}$ are calculated in a onesided manner everywhere, except for the derivatives $\phi_{x, x}^{0}$ and $\phi_{y, x}^{0}$ on $\Gamma_{I}$, which are calculated as the average of the derivative in the elements adjacent to this boundary from the left and from the right.

Thus the weak form is: find $\boldsymbol{u} \equiv\left\{u_{i}\right\} \in \mathcal{S}(\Omega)$ and $\phi^{m} \equiv\left\{\phi_{i}^{m}\right\} \in \mathcal{S}_{L}\left(\Omega_{L}\right), m=1, \ldots, P$, which satisfy the initial conditions (13), (14) in $\Omega$ and (61) in $\Omega_{L}$, and satisfy (82) and (83) for all $\boldsymbol{w}^{0} \equiv\left\{w_{i}\right\} \in \mathcal{S}(\Omega)$ and all $\boldsymbol{w}^{m} \equiv\left\{w_{i}^{m}\right\} \in \mathcal{S}_{L}\left(\Omega_{L}\right), m=1, \ldots, P$.

\subsection{Semi-discrete form}

We discretize the weak form described in the previous subsection in space using the standard Galerkin FE method. On the global level, the variables $u_{i}$ in $\Omega$ and $\phi_{i}^{m}$ in $\Omega_{L}$ are replaced 
by their finite-dimensional approximations

$$
\begin{aligned}
& u_{i}^{h}(\boldsymbol{x}, t)=\sum_{A \in \eta_{i}} d_{A i}^{0 h}(t) N_{A}(\boldsymbol{x}), \quad \boldsymbol{x} \in \Omega, i=x, y \\
& \phi_{i}^{m h}(\boldsymbol{x}, t)=\sum_{A \in \eta_{i}^{L}} d_{A i}^{m h}(t) N_{A}(\boldsymbol{x}), \quad \boldsymbol{x} \in \Omega_{L}, i=x, y, \quad m=1, \ldots, P
\end{aligned}
$$

Here $h$ is the mesh parameter, $A$ stands for the global node number, $\eta_{i}$ is the set of nodes in $\Omega$ on which there is no essential boundary condition prescribed on the displacement in the $i$ direction, $\eta_{i}^{L}$ is the set of nodes in $\Omega_{L}$ on which there are no essential boundary conditions prescribed on the displacement in the $i$ direction, $N_{A}$ is the global-level shape function associated with the variables $u_{i}$ in $\Omega$ and node $A$, or with the variables $\phi_{i}^{m}$ in $\Omega_{L}$ and node $A$, and $d_{A i}^{m h}$ is the value of $\phi_{i}^{m h}$ at node $A$. We take identical bilinear shape functions for the discretization of $u_{i}$ and for the discretization of all the $\phi_{i}^{M}$.

On the element level the analogous expansion is

$$
\begin{aligned}
& u_{i}^{e}(\boldsymbol{x}, t)=\sum_{a=1}^{N_{e n}} d_{a i}^{0 e}(t) N_{a}(\boldsymbol{x}), \quad \boldsymbol{x} \in \Omega^{e}, i=x, y \\
& \phi_{i}^{m e}(\boldsymbol{x}, t)=\sum_{a=1}^{N_{e n}} d_{a i}^{m e}(t) N_{a}(\boldsymbol{x}), \quad \boldsymbol{x} \in \Omega^{e}, i=x, y, \quad m=1, \ldots, P .
\end{aligned}
$$

Here $e$ stands for an element number, $N_{e n}$ is the number of element nodes, $a$ stands for the local element node number, $\Omega_{e}$ is the domain of element $e, N_{a}$ is the element-level displacement shape function associated with the node $a$, the quantities $d_{a i}^{m e}, m=0, \ldots, P$ are the values of $u_{i}^{e}$ and $\phi_{i}^{m e}$ at node $a$ of element $e$. We also write global expressions similar to (95) and (96) for the test functions $w_{i}^{m h}, m=0, \ldots, P$ and element level expressions similar to (97) and (98) for the element-level test functions $w_{i}^{m e}, m=0, \ldots, P$.

Substitution of the approximations (95) and (96) in the weak equations (82), (83) and (92)-(94) yields a system of ordinary differential equations in time, of the form

$$
\begin{aligned}
& \boldsymbol{M}_{0} \ddot{\boldsymbol{d}}+\boldsymbol{C}_{0} \dot{\boldsymbol{d}}+\boldsymbol{K}_{0} \boldsymbol{d}+\boldsymbol{G}_{0} \dot{\boldsymbol{\phi}}_{1}+\boldsymbol{H}_{0} \boldsymbol{\phi}_{1}=\boldsymbol{f} \\
& \boldsymbol{M}_{m} \ddot{\boldsymbol{\phi}}_{m}+\boldsymbol{C}_{m} \dot{\boldsymbol{\phi}}_{m}+\boldsymbol{K}_{m} \boldsymbol{\phi}_{m}+\boldsymbol{A}_{m} \dot{\boldsymbol{\phi}}_{m-1}+\boldsymbol{B}_{m} \boldsymbol{\phi}_{m-1}+\boldsymbol{G}_{m} \dot{\boldsymbol{\phi}}_{m+1}+\boldsymbol{H}_{m} \boldsymbol{\phi}_{m+1}=\mathbf{0}
\end{aligned}
$$

with $m=1, \ldots, P-1$;

$$
\boldsymbol{M}_{P} \ddot{\boldsymbol{\phi}}_{P}+\boldsymbol{C}_{P} \dot{\boldsymbol{\phi}}_{P}+\boldsymbol{K}_{P} \boldsymbol{\phi}_{P}+\boldsymbol{A}_{P} \dot{\boldsymbol{\phi}}_{P-1}+\boldsymbol{B}_{P} \boldsymbol{\phi}_{P-1}=\mathbf{0}
$$

with the initial conditions

$$
\begin{array}{lll}
\boldsymbol{d}^{i}(t=0)=\boldsymbol{d}_{0}^{i} ; & \dot{\boldsymbol{d}}^{i}(t=0)=\boldsymbol{v}_{0}^{i} ; & i=x, y \\
\boldsymbol{\phi}_{m}^{i}(t=0)=\mathbf{0} ; & \dot{\boldsymbol{\phi}}_{m}^{i}(t=0)=\mathbf{0} ; \quad i=x, y, \quad m=1, \ldots, P
\end{array}
$$


Here $\boldsymbol{d}$ and $\phi_{m}$ are the vectors whose entries are all the unknown nodal values of $u_{x}$ followed by all the unknown nodal values of $u_{y}$ in $\Omega$, and of $\phi_{m}^{x}$ followed by $\phi_{m}^{y}$ in $\Omega_{L}$, respectively; a dot indicates differentiation with respect to time.

The element level expressions may be extracted from (82), (83) and (92)-(94). The first (mass) and second (stiffness) terms in each of (82) and (83) contribute to the global mass and stiffness matrices respectively via the following arrays on the element level (we denote these arrays by the superscript $O M)$ :

$$
\begin{aligned}
& M_{0(a i)(b j)}^{O M e}=\delta_{i j} \rho \int_{\Omega^{e}} N_{a} N_{b} d \Omega \\
& K_{0(a i)(b j)}^{O M e}=\int_{\Omega^{e}} N_{a, k} C_{i k j l} N_{b, l} d \Omega .
\end{aligned}
$$

The arrays $M_{0}^{O M e}$ and $K_{0}^{O M e}$ correspond to the first and second terms in (82). The expressions corresponding to $M_{m}^{O M e}$ and $K_{m}^{O M e}(m=1, \ldots, P)$ the first and second terms in (83) are similar, aside for the integration which takes place in an element in $\Omega_{I}$ rather than in $\Omega$.

In the following expressions we use the notation $c_{i j}^{*}$ and $c_{i j}^{* *}$ as was defined in (56). The contribution from the $B_{I}^{m}$ term (denoted by the superscript $B I$ ) to the damping matrices is $(m=1, \ldots, P)$ is

$$
\begin{aligned}
& C_{m(a i)(b j)}^{B I e}=\frac{c_{i j}^{* *}}{c_{L}} a_{m-1} \int_{\Gamma_{I}} N_{a} N_{b} d \Gamma \\
& A_{m(a i)(b j)}^{B I e}=-\frac{c_{i j}^{* *}}{c_{L}} a_{m-1} \int_{\Gamma_{I}} N_{a} N_{b} d \Gamma
\end{aligned}
$$

The contribution from the $B_{I}^{m}$ term to the stiffness matrixes is

$$
\begin{aligned}
& K_{m(a i)(b j)}^{B I e}=-c_{i j}^{* *} \beta \int_{\Gamma_{I}} N_{a} N_{b, y} d \Gamma+c_{i j}^{*} \int_{\Gamma_{I}} N_{a} N_{b, y} d \Gamma \\
& B_{m(a i)(b j)}^{B I e}=-c_{i j}^{* *} \beta \int_{\Gamma_{I}} N_{a} N_{b, y} d \Gamma-c_{i j}^{* *} \int_{\Gamma_{I}} N_{a} N_{b, x} d \Gamma,
\end{aligned}
$$

Similarly, the contribution from the $B_{E}^{m}$ term (denoted by the superscript $B E$ ) to the damping matrices is $(m=0, \ldots, P-1)$

$$
\begin{aligned}
G_{m(a i)(b j)}^{B E e} & =-\frac{c_{i j}^{* *}}{c_{L}} a_{m} \int_{\Gamma_{E}} N_{a} N_{b} d \Gamma \\
C_{m(a i)(b j)}^{B E e} & =\frac{c_{i j}^{* *}}{c_{L}} a_{m} \int_{\Gamma_{E}} N_{a} N_{b} d \Gamma
\end{aligned}
$$

and from the LK termination,

$$
C_{P(a i)(b j)}^{B E e}=\rho s_{i j}^{L K} \int_{\Gamma_{E}} N_{a} N_{b} d \Gamma
$$


The contribution from the $B_{E}^{m}$ term to the stiffness matrix is

$$
\begin{aligned}
& K_{m(a i)(b j)}^{B E e}=-c_{i j}^{* *} \beta \int_{\Gamma_{E}} N_{a} N_{b, y} d \Gamma-c_{i j}^{*} \int_{\Gamma_{E}} N_{a} N_{b, y} d \Gamma \\
& H_{m(a i)(b j)}^{B E e}=-c_{i j}^{* *} \beta \int_{\Gamma_{E}} N_{a} N_{b, y} d \Gamma+c_{i j}^{* *} \int_{\Gamma_{E}} N_{a} N_{b, x} d \Gamma .
\end{aligned}
$$

The different contributions (denoted $O M, B I$ and $B E$ ) to the matrices $\boldsymbol{M}, \boldsymbol{C}, \boldsymbol{K}, \boldsymbol{A}, \boldsymbol{B}$, $\boldsymbol{G}$ and $\boldsymbol{H}$ are summed, and the resulting matrices are assembled by the standard FEM assembly method, to form the system (99)-(103).

\subsection{Computational aspects}

\subsubsection{Parameters of the ABC}

The parameters $a_{m}$ in the recursive relations (64) and (65) allow some freedom in the formulation of the absorbing layer, which may influence the stability and accuracy properties of the scheme. As noted in [15], small values of this parameter correspond to a scalar boundary condition that is adapted for incident waves arriving at grazing incidence but is unfortunately unstable. In general, we adhere to the choice of $a_{m}=1$, which, under the conditions specified herein, is stable. Note that for a high DAB order $P$, the total reflection coefficient is the product of $\mathrm{P}$ basic reflection coefficients, so even though the choice $a_{m}=1$ may give a basic reflection coefficient which is close to unity for oblique waves, the product of all these reflection coefficients may be arbitrarily small. However, as noted above, long-time accuracy typically requires the addition of terms optimized for evanescent waves.

\subsubsection{Discretization in Time}

The system (99)-(101) was solved using the implicit Newmark method, as done previously in [15], with the same stabilizing modification of the Newmark parameters that was used in the isotropic and acoustic cases. Namely, the standard Newmark parameters $\beta=0.25$ and $\gamma=0.5$ were modified to $\beta_{L}=0.36, \gamma_{L}=0.7$ inside the layer and along a single additional row of elements in $\Omega_{I}$ which is adjacent to $\Gamma_{I}$. The reason for this is that in this row of elements we calculate the derivative $u_{i, x}$ and use it in calculations performed in the layer. Throughout the rest of the domain $\Omega_{I}$, we take the standard values of $\beta_{L}=0.25, \gamma_{L}=0.5$.

\subsubsection{Stability}

We now describe the stability properties of the DAB for anisotropic elastodynamics at the various levels: the continuous level, the semi-discrete level (i.e., after space discretization using FEs), and the fully discrete level (i.e., after space and time discretization).

At the continuous level, we believe the DAB for elastodynamics to be stable, although we currently do not have a full stability proof. In [19] stability was proved for an elastodynamics 
formulation with a single artificial boundary, using the same high-order ABC that the DAB in the present paper is based on. Although [19] dealt with the isotropic case, the stability proof does not make direct use of the isotropy, and thus remains valid for the anisotropic case. The proof does not require any special treatment of inverse modes, just as the proof of stability of the LK-like condition (see Section 2.4) holds true for any kind of waves and anisotropic medium. However, the proof in [19] does not cover the case where the single $\mathrm{ABC}$ on $\Gamma_{E}$ is replaced by a DAB layer, as in the present work. In [14], a stability proof is provided for a configuration with a DAB, but for the scalar case (acoustics) only.

To determine stability at the semi-discrete and fully-discrete levels, we solve the associated eigenvalue problems. We start by considering the semi-discrete problem. We rewrite the system (99)-(101) as a general second order system, i.e.,

$$
\hat{M} \ddot{\boldsymbol{u}}+\hat{\boldsymbol{C}} \dot{\boldsymbol{u}}+\hat{\boldsymbol{K}} \boldsymbol{u}=\hat{\boldsymbol{f}}
$$

where $\hat{\boldsymbol{M}}$ is the global mass matrix which includes the $\boldsymbol{M}_{m}$ as submatrices, $\hat{\boldsymbol{C}}$ is the global damping matrix which includes $\boldsymbol{C}_{m}, \boldsymbol{A}_{m}$, and $\boldsymbol{G}_{m}$ as submatrices, and $\hat{\boldsymbol{K}}$ is the global stiffness matrix which includes $\boldsymbol{K}_{m}, \boldsymbol{B}_{m}$, and $\boldsymbol{H}_{m}$ as submatrices. The stability of this semidiscrete system is determined from the solution of the corresponding quadratic eigenvalue problem,

$$
\left(\hat{\Lambda}^{2} \hat{\boldsymbol{M}}+\hat{\Lambda} \hat{\boldsymbol{C}}+\hat{\boldsymbol{K}}\right) \boldsymbol{u}=\mathbf{0}
$$

The condition for stability is that all the eigenvalues $\hat{\Lambda}$ satisfy $\operatorname{Re}(\hat{\Lambda}) \leq 0$.

Now we consider the fully-discrete scheme. The Newmark time-stepping method applied to (115) yields [42] the algebraic system

$$
\boldsymbol{M}^{*} \boldsymbol{u}_{n+1}+\boldsymbol{C}^{*} \boldsymbol{u}_{n}+\boldsymbol{K}^{*} \boldsymbol{u}_{n-1}+\Delta t^{2} \boldsymbol{f}_{n}^{*}=\mathbf{0}
$$

where

$$
\begin{aligned}
& \boldsymbol{M}^{*}=\hat{\boldsymbol{M}}+\gamma \Delta t \hat{\boldsymbol{C}}+\beta \Delta t^{2} \hat{\boldsymbol{K}} \\
& \boldsymbol{C}^{*}=-2 \hat{\boldsymbol{M}}+(1-2 \gamma) \Delta t \hat{\boldsymbol{C}}+(1 / 2-2 \beta+\gamma) \Delta t^{2} \hat{\boldsymbol{K}} \\
& \boldsymbol{K}^{*}=\hat{\boldsymbol{M}}-(1-\gamma) \Delta t \hat{\boldsymbol{C}}+(1 / 2+\beta-\gamma) \Delta t^{2} \hat{\boldsymbol{K}}
\end{aligned}
$$

Here $\boldsymbol{u}_{n}$ is the value of $\boldsymbol{u}$ at time step $n$, and $\boldsymbol{f}_{n}^{*}$ is the expression for the external force vector at time step $n$. Stability is determined from the quadratic eigenvalue problem

$$
\left(\Lambda^{* 2} \boldsymbol{M}^{*}+\Lambda^{*} \boldsymbol{C}^{*}+\boldsymbol{K}^{*}\right) \boldsymbol{u}=\mathbf{0}
$$

The condition for stability is that all the eigenvalues $\Lambda^{*}$ satisfy $\left|\Lambda^{*}\right| \leq 1$.

In order to determine the stability of the semi-discrete and fully discrete formulations, for the DAB scheme described above, we solved numerically the eigenvalue problems (116) and (121), respectively, for various choices of the computational parameters. In each case, 
we checked if the stability criteria written above were satisfied. In addition, we ran the corresponding time-dependent simulations for a very large number of time-steps, further verifying the stability results obtained from the fully-discrete eigenvalue analysis. All this yielded the following results. The semi-discrete formulation is long-time unstable, namely there are eigenvalues of (116) with a small positive real part. However, the fully-discrete problem is stable provided some conditions outlined below are met. Thus, the dissipative time discretization that we employ allows us to regain stability at the fully-discrete level, after it was lost at the semi-discrete level.

Similarly to the isotropic case discussed in [15], the scheme loses its long-time stability in the following situations.

1. When the material properties support inverse modes, the scheme becomes unstable in the presence of such waves. As we noted above, the developed boundary condition does not account for the presence of such modes on the boundary. We also illustrate this instability in the numerical experiments below.

2. Replacing of the periodic boundary conditions on the north and south boundaries by traction-free conditions, or by some combination of fixed and traction-free boundary conditions, destabilizes the scheme.

3. Even though the proposed scheme is fully implicit, it was found that in order to maintain long-time stability, the value of the CFL number must lie in a certain finite interval. The CFL number is defined as $c_{L} \Delta t / h$, where $h$ is the mesh parameter, namely a characteristic element size, and $c_{L}$ is the maximal wave velocity of the material. Thus, if the mesh is refined or coarsened, the time step may have to be adjusted accordingly, to keep the CFL in the interval of stability. The simplest safe practice is to choose a CFL number in the stable range, and keep it constant under any changes in the discretization. The mesh parameter and/or time-step size have also to be adjusted when the material properties are changed, again to keep the CFL number in the allowed range. It should be remarked that when the implicit time-stepping is replaced by an explicit Newmark time-stepping, which is non-dissipative, the scheme becomes unstable for any value of the CFL number.

Fig. 4 depicts the allowable range of the CFL number, as tested with a small mesh of $4 \times 4$ elements in the interior domain plus $4 \times 4$ elements in the DAB layer. The element size was fixed as 0.05, and the time step was varied. The materials are the same orthotropic materials specified in the "Numerical Examples" section below. The allowable (stable) zone for a particular material is shown in Fig. 4 as the area between the plotted lower and upper limits of stability. In principle, the eigenvalue analysis of the fully discrete scheme determines both the upper and lower limits of stability. However, we have used this analysis to determine the lower limit, while in the case of the upper limit, the eigenvalues were extremely close to 1, either from above or from below, and thus it was difficult to determine the onset of instability. Instead, the upper 


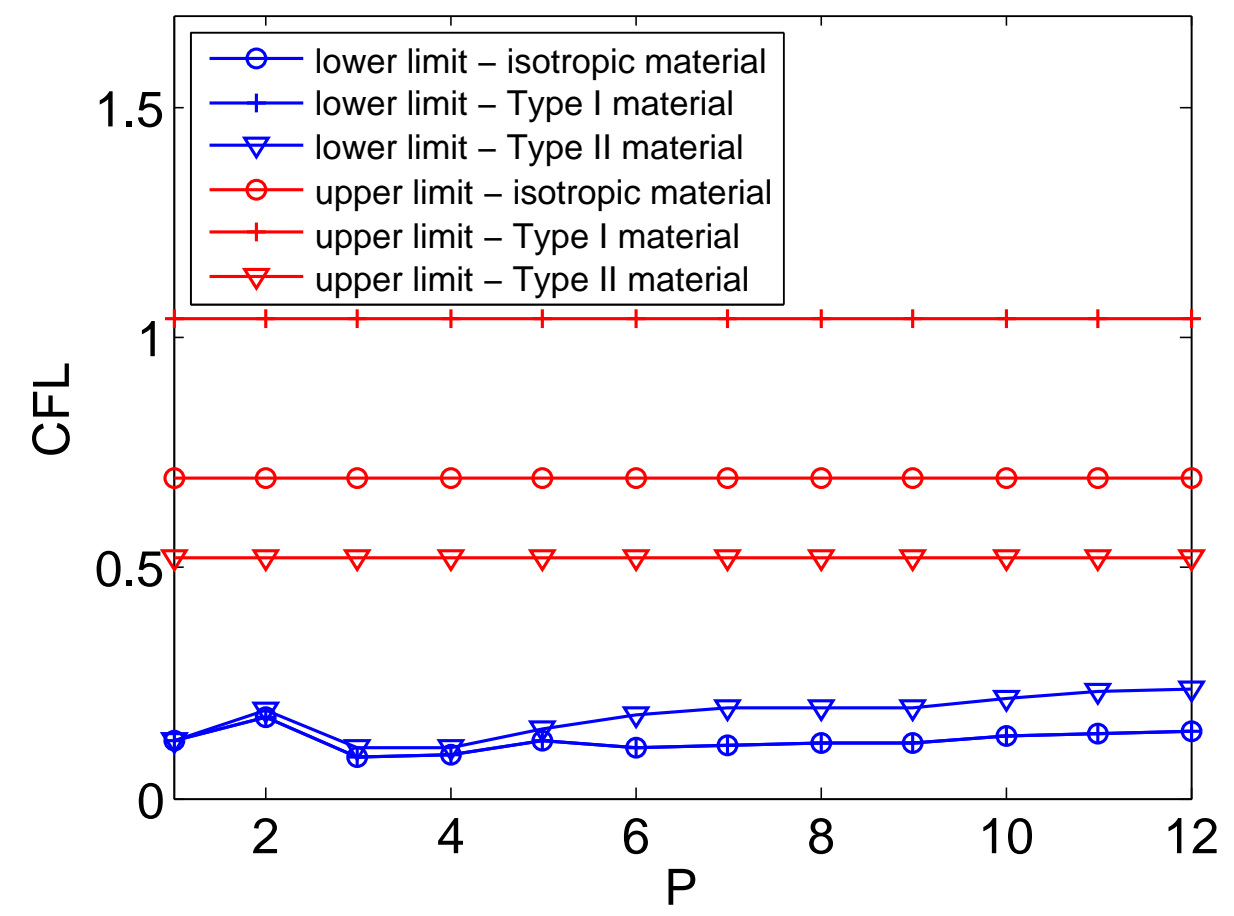

Figure 4: Stability limits, small problem, different types of materials

stability limit was determined by running the problem for a long time (200,000 time steps), and checking for solution divergence.

It should be noted that for values slightly outside the stability limits the divergence occurs only for very long run times, and therefore in practice the discretization requirements may be relaxed somewhat if the simulation is run for much shorter periods of time.

\subsubsection{Accuracy}

The accuracy of the DAB method in the elastic anisotropic setting was measured numerically by comparing the solution in the truncated waveguide to a reference solution $\boldsymbol{u}^{h, r e f}$. The latter was constructed by extending the waveguide so that in the reference model the truncation boundary is located much farther from the original interior domain $\Omega_{I}$, so that waves reflected from that far truncation boundary do not reach back to $x=x_{E}$ during the simulation time. Thus, the difference between the solution $\boldsymbol{u}^{h}$ computed in $\Omega$ and the reference solution $\boldsymbol{u}^{h, r e f}$ is approximately the DAB error. The error measure is therefore

$$
E=\frac{\left\|\boldsymbol{u}^{h}-\boldsymbol{u}^{h, r e f}\right\| \|_{\Omega_{I} \times[0, T]}}{\left\|\boldsymbol{u}^{h, r e f}\right\|_{\Omega_{I} \times[0, T]}},
$$


where $\|\cdot\|_{\mathcal{M}}$ is the $l_{2}$ norm calculated on the manifold $\mathcal{M}$, and $T$ is the simulation time. Note that the errors are not measured inside $\Omega_{L}$.

Additionally, we use the following normalized error norm in space for plotting the error in space as a function of time:

$$
e(t)=\frac{\left\|\boldsymbol{u}(t)^{h}-\boldsymbol{u}(t)^{h, r e f}\right\|_{\Omega_{I}}}{\sqrt{A}} .
$$

Here $A$ is the area of the domain $\Omega_{I}$.

These measures will be used to asses the accuracy in the numerical examples that follow.

\section{Numerical Examples}

\subsection{Modeled materials}

In the paper [27], the authors classify materials of general anisotropic properties into four groups, based on their slowness curves:

- Isotropic materials.

- Type I materials - anisotropic materials with a convex slowness curve.

- Type II materials, which are anisotropic, have a non-convex slowness curve, but support no inverse modes.

- Type III materials, which are anisotropic, have a non-convex slowness curve, and support inverse modes.

Using the PML method proposed there it was found that materials up to type II give stable results, while the inverse modes introduced by Type III materials result in instabilities.

Here we shall examine the performance of the DAB method using a material from each of these types, as described below.

- Isotropic: $c_{11}=3, c_{22}=3, c_{12}=1, c_{33}=1$

- Type I: $c_{11}=3, c_{22}=2, c_{12}=0.25, c_{33}=1$

- Type II: $c_{11}=3, c_{22}=3, c_{12}=0.25, c_{33}=0.6$

- Type III: $c_{11}=3, c_{22}=3, c_{12}=0.6, c_{33}=2$

The corresponding slowness curves of these materials are shown below in Fig. 5

Note that $c_{11}$ is similar in all of the cases, and therefore the mesh and time step used in all the materials are identical in all the cases shown below. 


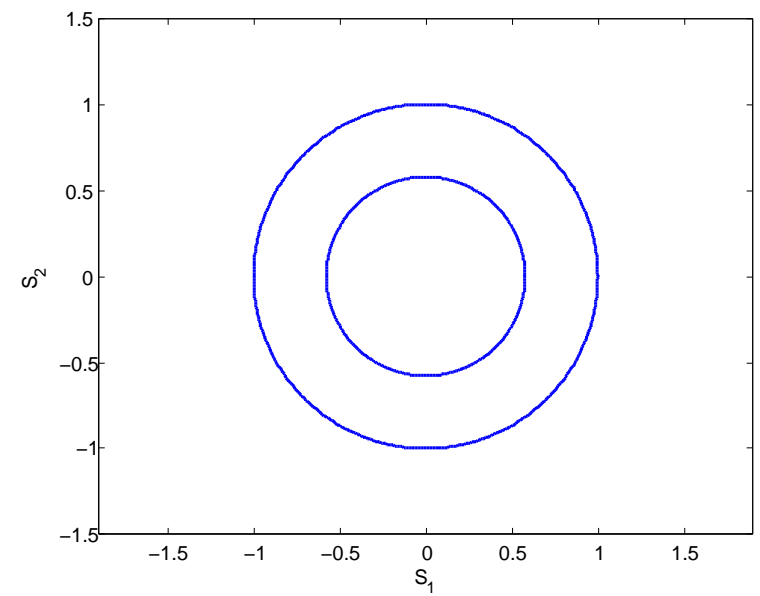

(a)

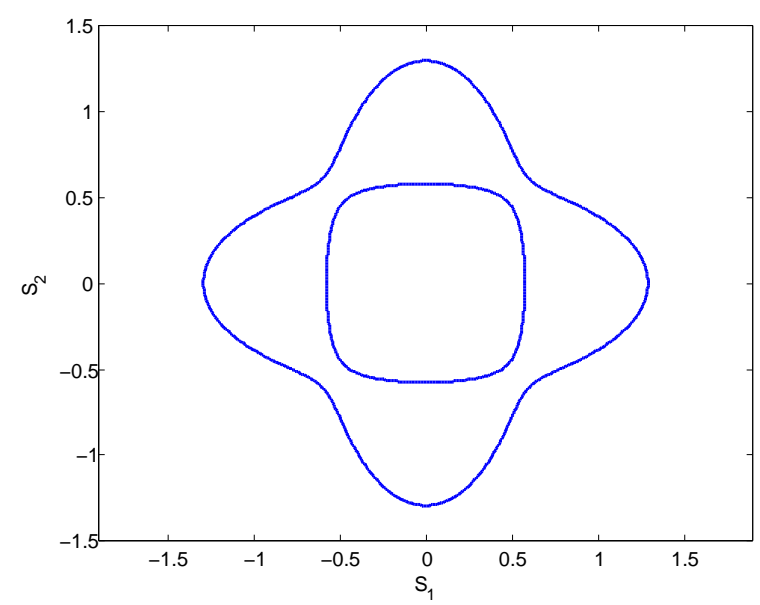

(c)



(b)

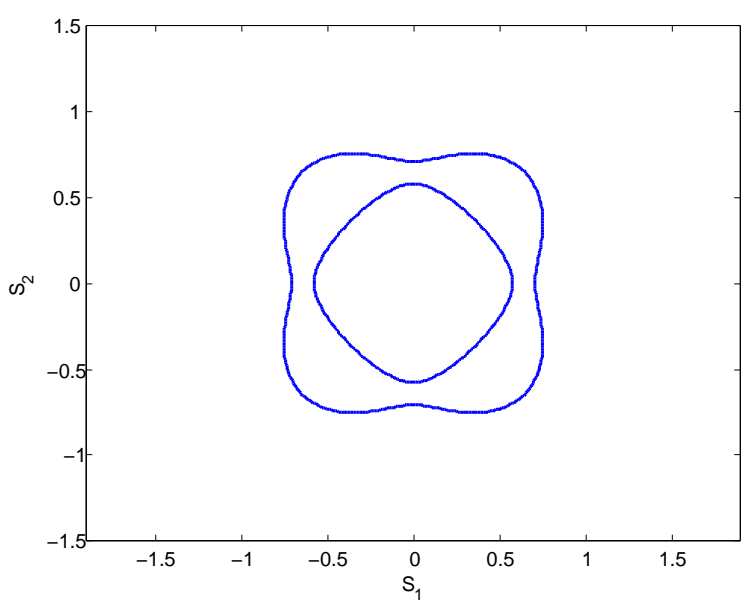

(d)

Figure 5: Slowness diagrams of the different materials: (a) Isotropic; (b) Anisotropic Type I; (c) Anisotropic Type II; (d) Anisotropic Type III.

\subsection{Rate of convergence in space and time}

The errors generated by the DAB scheme, with respect to the original unbounded-domain problem, are of two types: discretization errors and domain truncation errors. We will investigate the latter errors in later subsections. In this subsection we would like to verify that the DAB formulation does not harm the rate of convergence in space and time of the FE scheme. With bilinear quadrilateral elements and using Newmark time-stepping (see Section 4.3.2), the optimal rate of convergence of the standard FE formulation is known to be 2 in both space and time. To determine the rate of convergence of the DAB scheme, we apply the following procedure.

We take a hierarchy of three meshes, with mesh parameters $h_{0}, h_{0} / 2$ and $h_{0} / 4$, and three 
time-step sizes, i.e., $\Delta t_{0}, \Delta t_{0} / 2$ and $\Delta t_{0} / 4$. For each combination of these parameters, we solve the same test problem with the DAB scheme. We use the notation $u(h, \Delta t ; \boldsymbol{x}, t)$ for the numerical solution obtained from the DAB scheme using the parameters $h$ and $\Delta t$. For clarity, we shall omit the dependence on $\boldsymbol{x}$ and $t$ in the sequel, and thus simply denote the solution by $u(h, \Delta t)$. We define $u_{\text {ref }}=u\left(h_{0} / 4, \Delta t_{0} / 4\right)$, and regard this as a reference solution, since it is obtained with the finest discretization in space and time.

Now we define the following space and time error measures:

$$
E_{s}(h)=\left\|u(h, \Delta t / 4)-u_{r e f}\right\| \quad, \quad E_{t}(\Delta t)=\left\|u(h / 4, \Delta t)-u_{r e f}\right\|
$$

where the $\|\cdot\|$ is the $L_{2}$ norm in space and time. We make use of the error estimate (see, e.g., [42])

$$
E_{s}(h) \leq C_{s} h^{p} \quad, \quad E_{t}(\Delta t) \leq C_{t}(\Delta t)^{q}
$$

where $p$ are $q$ are, respectively, the (unknown) rates of convergence in space and time. Concentrating first on the space convergence, and replacing the inequalities in (125) by approximate equalities, we have

$$
E_{s}\left(h_{0}\right) \simeq C_{s} h_{0}^{p} \quad, \quad E_{s}\left(h_{0} / 2\right) \simeq C_{s}\left(h_{0} / 2\right)^{p}
$$

from which we deduce

$$
E_{s}\left(h_{0}\right) / E_{s}\left(h_{0} / 2\right)=2^{p} .
$$

Taking the log on both sides and rearranging yields the formula

$$
p=\frac{\log \left[E_{s}\left(h_{0}\right) / E_{s}\left(h_{0} / 2\right)\right]}{\log 2}
$$

for the rate of convergence in space. Similarly, we get

$$
q=\frac{\log \left[E_{t}\left(\Delta t_{0}\right) / E_{t}\left(\Delta t_{0} / 2\right)\right]}{\log 2}
$$

for the rate of convergence in time.

We applied this procedure to the test problem described in the next section, for the various anisotropic materials, with $h_{0}=0.05$ (which corresponds to a mesh of $60 \times 200=12000$ elements) and $\Delta t_{0}=0.005$. Formulas (128) and (129) yielded rates of convergence in space and time which are remarkably close to the optimal rate of 2 , with up to $1 \%$ deviation. Repeating this calculation with a simple LK condition replacing the DAB layer yielded the same result. This establishes that the DAB construction does not affect the convergence of the standard FE formulation with increasing discretization levels. 


\subsection{Initial pulse example}

The discrete FEM scheme of the DAB method (the present example as well as the subsequent ones) was tested on a numerical setup consisting of a waveguide with the dimensions $b=3$, $x_{I}=10$. The DAB layer extended $n_{L}$ elements beyond $x_{E}$, where $n_{L}$ varied, the total length of the waveguide was therefore $x_{E}=x_{I}+h \cdot n_{L}$. In all our experiments we took $\rho=1$, with the elastic properties taken according to the material type, as described in the previous subsection.

We built two meshes for the numerical experiments: Mesh A in $\Omega_{I}$ was composed of $60 \times 200$ square elements, where the side length of each square element was $h=0.05$, with $n_{L}=4$ elements across the width of $\Omega_{L}$. The time step used with Mesh A was taken as $\Delta t=0.005$.

Mesh B was composed of $30 \times 100$ square elements with a side length of $h=0.1$ in $\Omega_{I}$, with a layer made of the same elements, with $n_{L}=2$. The time step used with mesh $\mathrm{B}$ was $\Delta t=0.01$.

The following $C_{1}$-continuous initial conditions were set in the waveguide:

$$
\begin{aligned}
& u_{x 0}(x, y)= \begin{cases}{\left[(x-8.5)^{2}-1\right]^{2} \sin \left(\frac{2 \pi y}{b}\right)} & \text { for } 7.5 \leqslant x \leqslant 9.5 \\
0 & \text { otherwise }\end{cases} \\
& u_{y 0}(x, y)=0 \\
& v_{x 0}(x, y)=0 \\
& v_{y 0}(x, y)=0 .
\end{aligned}
$$

All subsequent runs were made with mesh A, unless stated otherwise.

When examined for stability, the isotropic materials and the materials of Types I and II gave stable results when run within the stable CFL window (see Fig. 4), while the Type III material gave unstable results for all conditions. The ustable behavior of the material of Type III closely follows the results obtained using the PML-based scheme described in [27].

The scheme was tested for accuracy by running it with the three stable material types for different values of $P$ and $n_{L}$, and comparing the results to a waveguide 2.5 times longer than $x_{E}$, which was sufficient in all cases for the wave not to be reflected back to the location of the tested truncation boundary $x_{E}$. The results are shown in Fig. 6. Unless stated otherwise, the duration of the simulation was taken as $T=10$.

The accuracy plot shows that the DAB scheme succeeds in absorbing the outgoing waves for the three materials shown. When compared to the isotropic material, the behavior of the orthotropic material of Type I is quite similar, while the performance of the Type II orthotropic material is slightly improved: the error decreases faster with $P$, and settles at the discretization error at about $P=4$, compared with $P=6$ for the other two materials. This suggests that the concavity of the slowness curve is not in itself a shortcoming as far as the DAB layer is concerned.

Fig. 7 shows the normalized $L_{2}$ error in space as a function of time for the three stable 


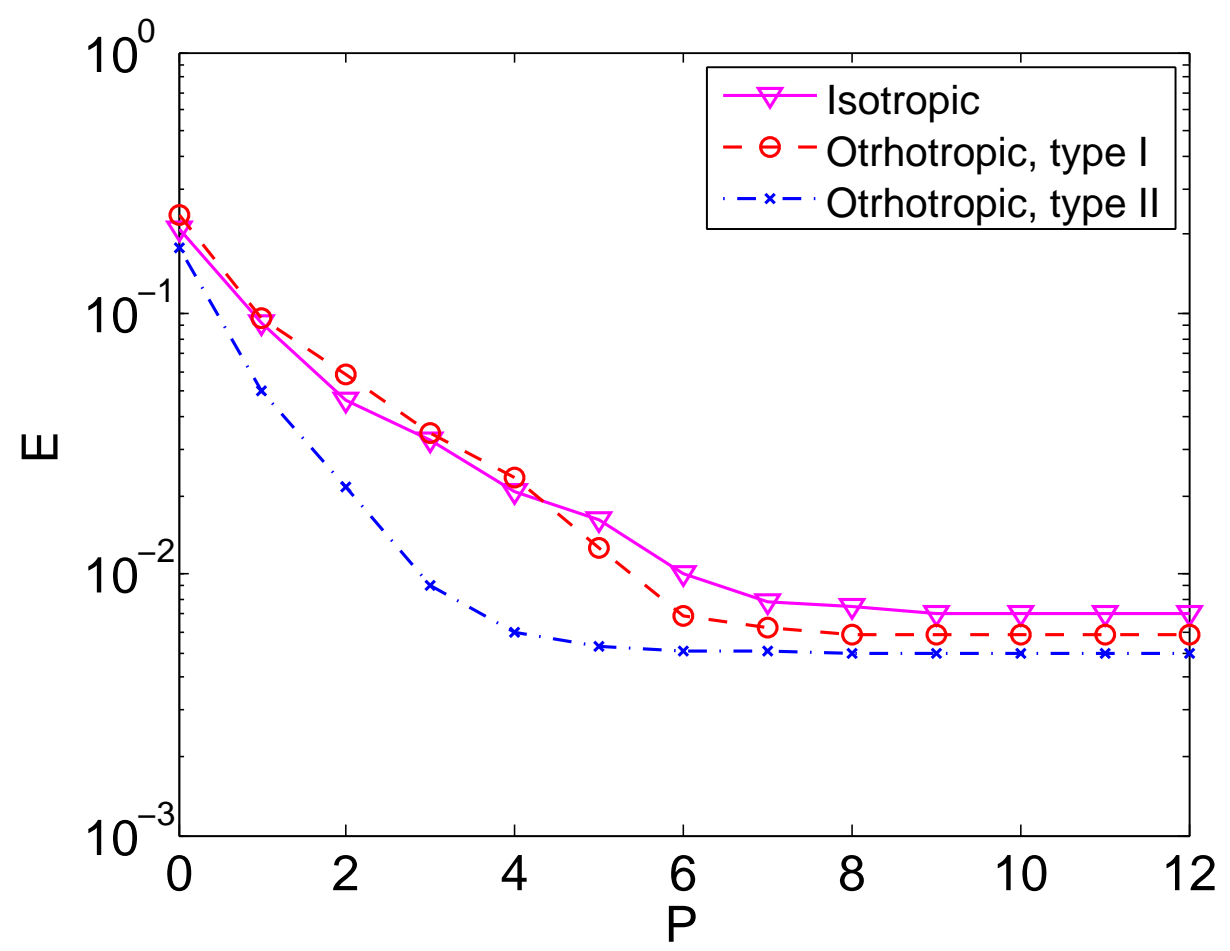

Figure 6: Accuracy of the stable DAB scheme for periodic boundary conditions, Mesh A

materials, and for 4 representative values of $P$. The graphs show the gradual improvement of the accuracy with $P$ in all 3 cases, and similar to Fig. 6 , it can be seen that the material of Type II converges faster with $P$ than the other materials.

In Figs. 8-10 we show snapshots of the solution in a waveguide with periodic North and south boundary conditions at different times for $P=5$ in the isotropic material, and in materials of Type I and II. In addition, in Fig. 11 we show snapshots of the type III material for $P=0$, which is stable in this low order. The $\Gamma_{I}$ boundary is marked in red on the snapshots.

It can be seen that the contour patterns of the isotropic, Type I and Type II materials are initially quite similar, and the particular features of each material type become pronounced as the simulation progresses. The patterns of the type III material, however, differ from the other materials from the outset. All the materials retain the antisymmetric structure of the initial conditions throughout the simulation.

The low order condition in the Type III material results in differences between the fields in the reference waveguide and in the truncated solution waveguide. Also, one can can see that the pulse source actually undergoes apparent contraction in the material of Type III (see $11 \mathrm{c}$ and $11 \mathrm{e}$ ).

To check the effect of the mesh on the results, we repeated the initial pulse run, with 


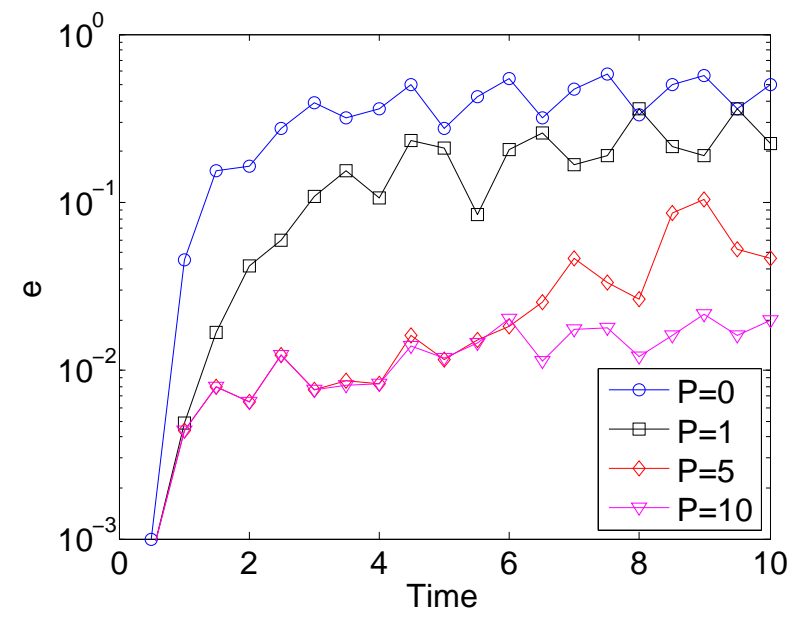

(a)

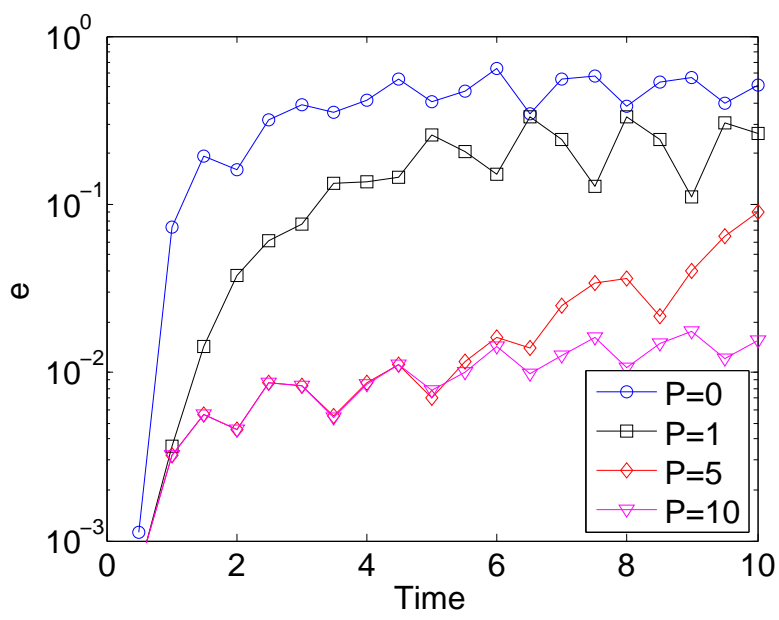

(b)

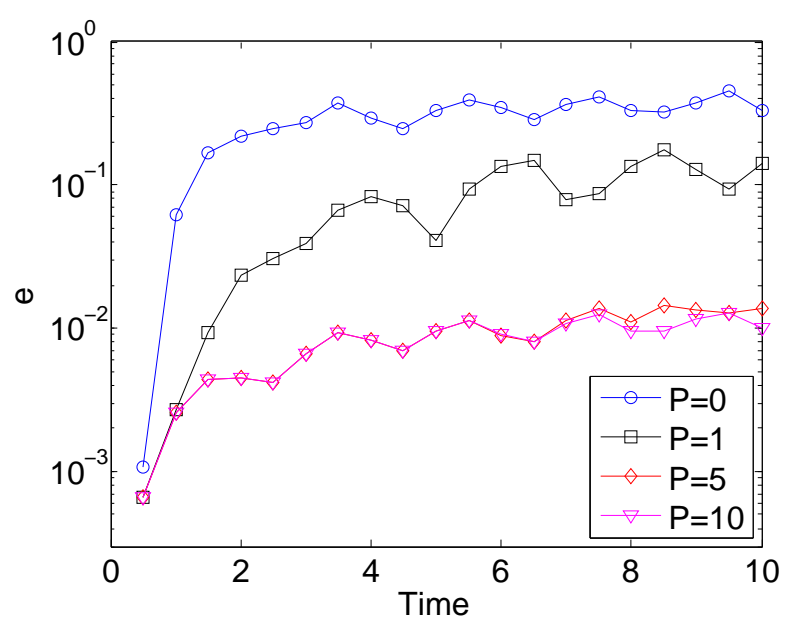

(c)

Figure 7: Evolution of the $L_{2}$ error in time for 3 different materials: (a) Isotropic; (b) Anisotropic Type I; (c) Anisotropic Type II

Mesh A replaced by Mesh B, the resulting errors as a function of $P$ are shown in Fig. 12.

The error levels reach the discretization-error saturation values earlier in this mesh - at $P=4$ for the isotropic material, at $P=5$ for the Type I material, and at $P=3$ in the Type II material. The general appearance of the graphs is similar to that of the graphs for the finer mesh, aside for the reduced accuracy.

When compared with Fig. 6, the discretization error levels are larger by a factor of $2-2.5$. This is lower than the theoretical convergence rate which dictates a factor of 4 upon transition from the coarse mesh to the twice-finer one. This may perhaps be explained by the part of the discretization error associated with the DAB scheme itself, which may be slower than the rate expected from the FE discretization.

In order to examine the effect of the simulation time on the error as a function of the 



(a)

(b)
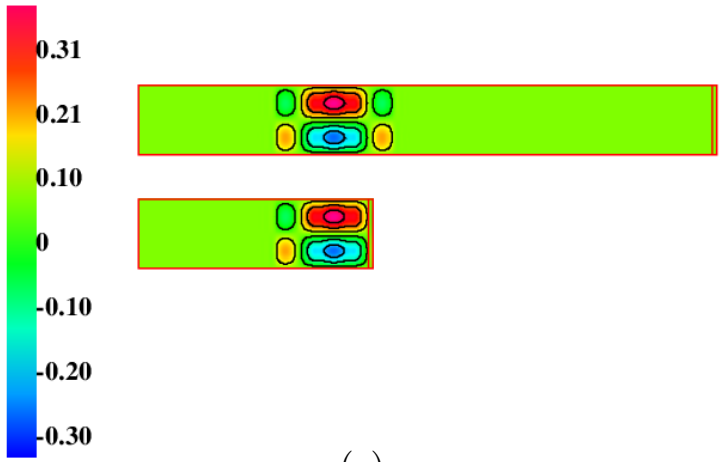

(c)
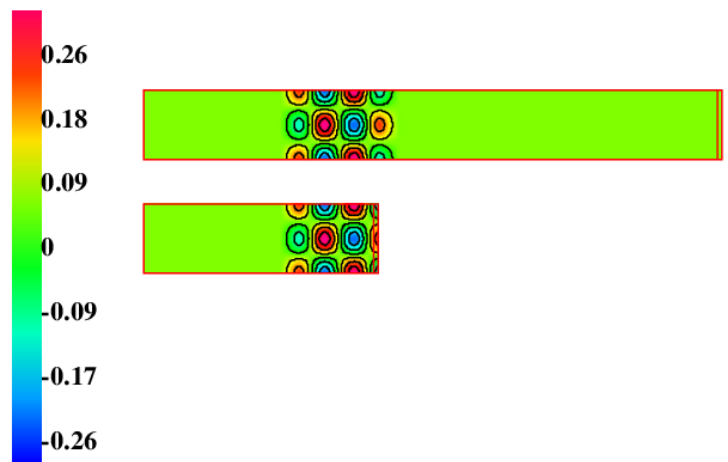

.09

17

.26

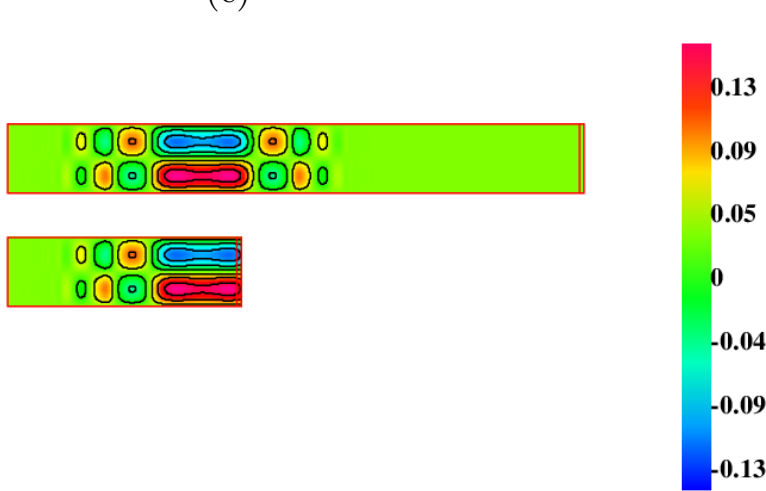

(e)
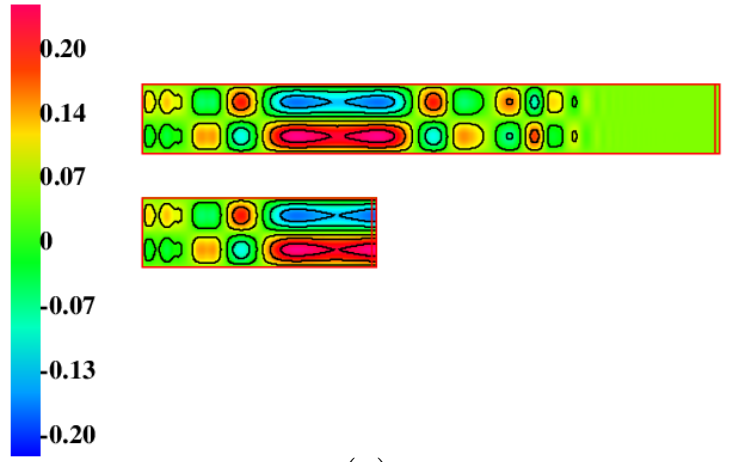

(g)

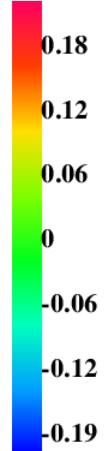

(d)
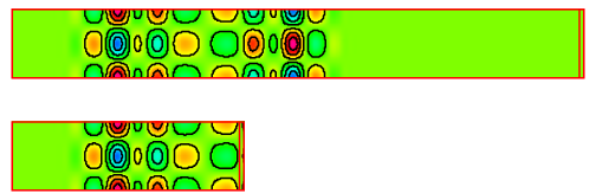

\section{(f)}

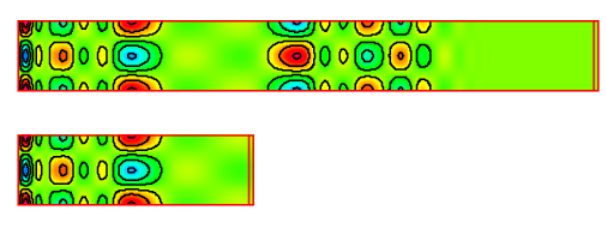



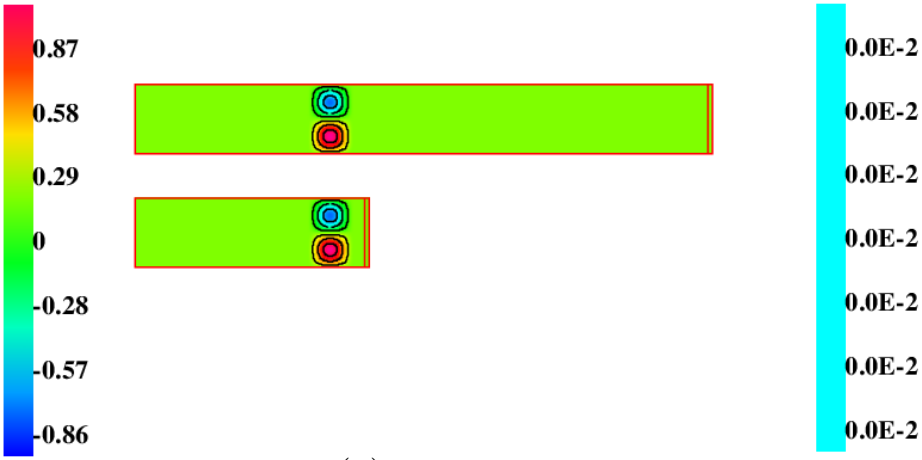

(a)

(b)
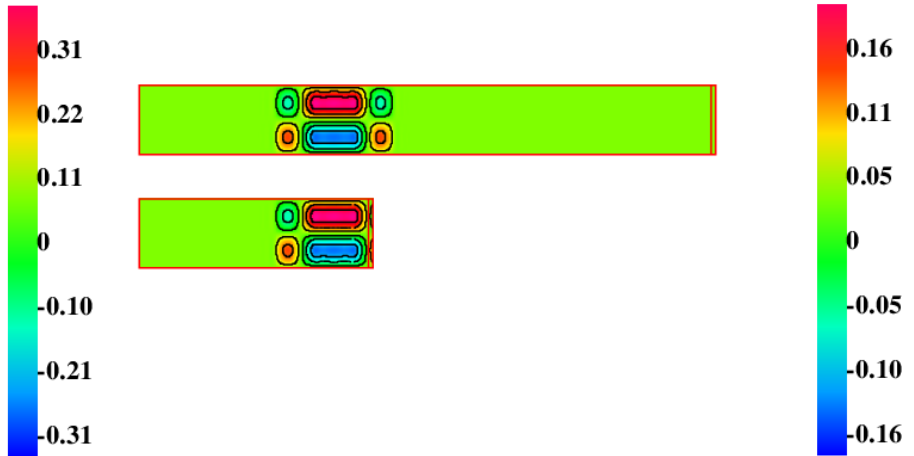

(c)

(d)
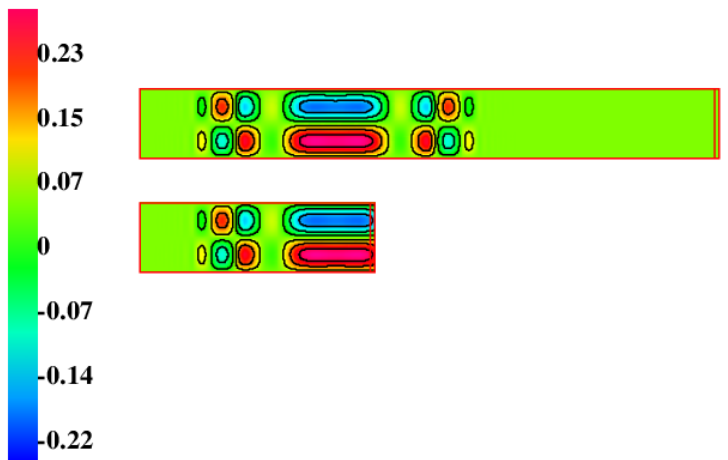





(e)
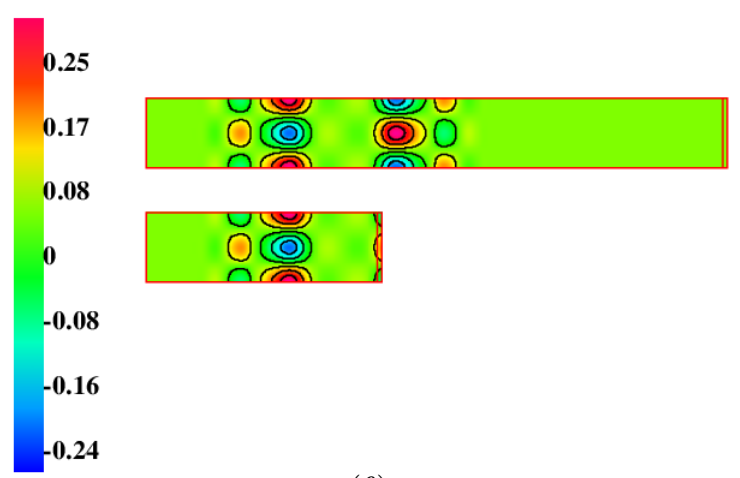

(f)
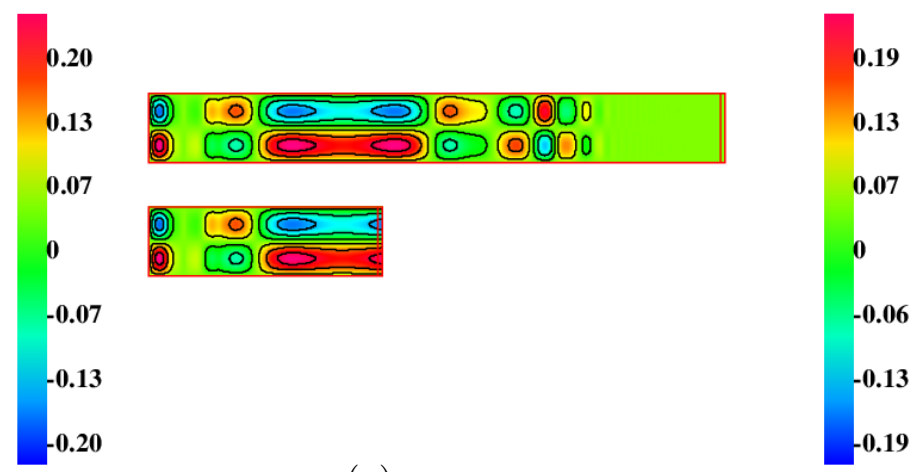

(g)

(h)

Figure 9: Pulse propagation in waveguide, material Type I: snapshots of solution: (a)-(b) $u_{x}$ and $u_{y}$ at $t=0$; (c)-(d) $u_{x}$ and $u_{y}$ at $t=1$; (e)-(f) $u_{x}$ and $u_{y}$ at $t=3$; (g)-(h) $u_{x}$ and $u_{y}$ at $t=6$. 

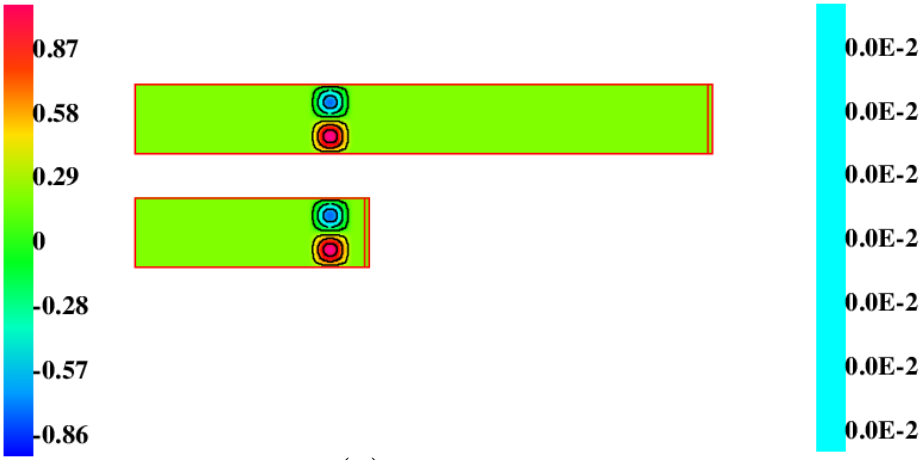

(a)

(b)
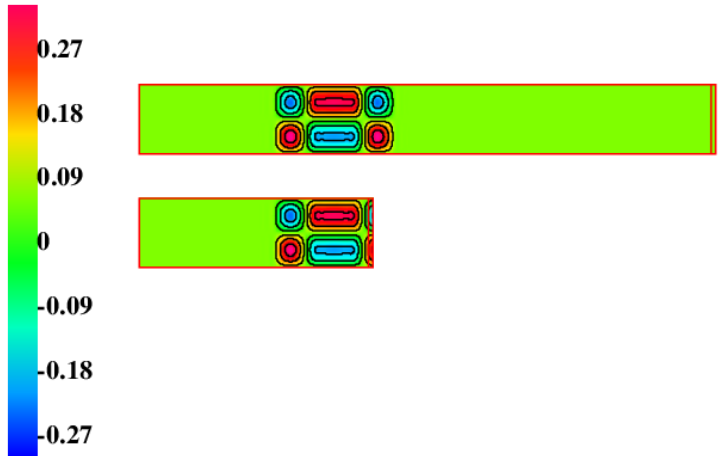

(c)
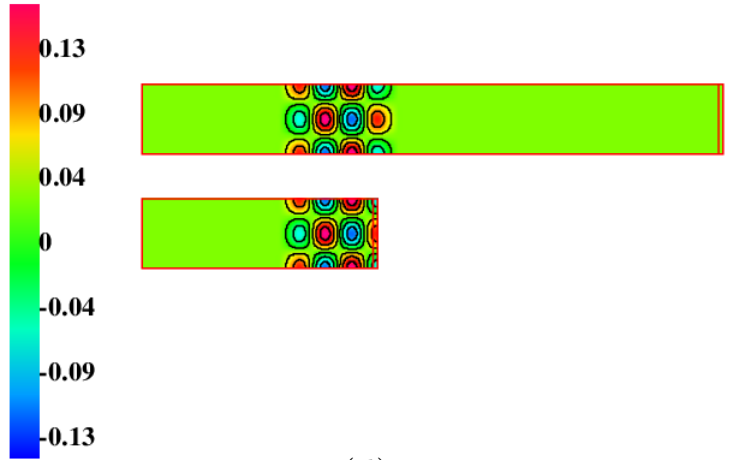

04

09

0.13

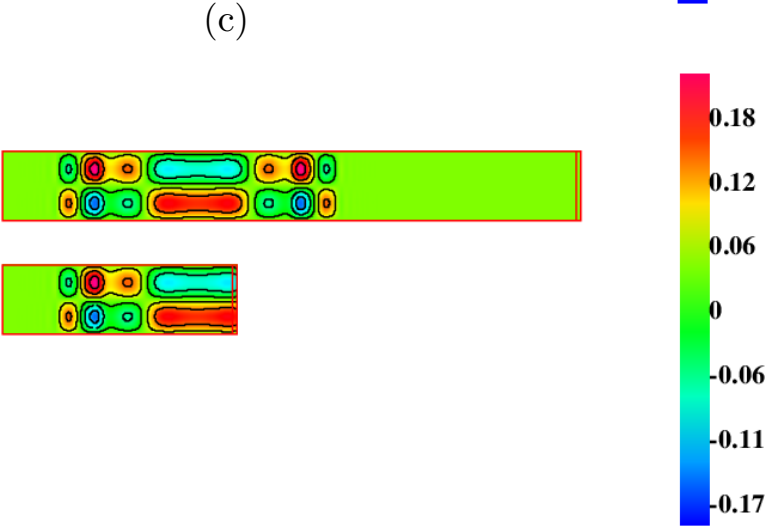

(e)

(f)
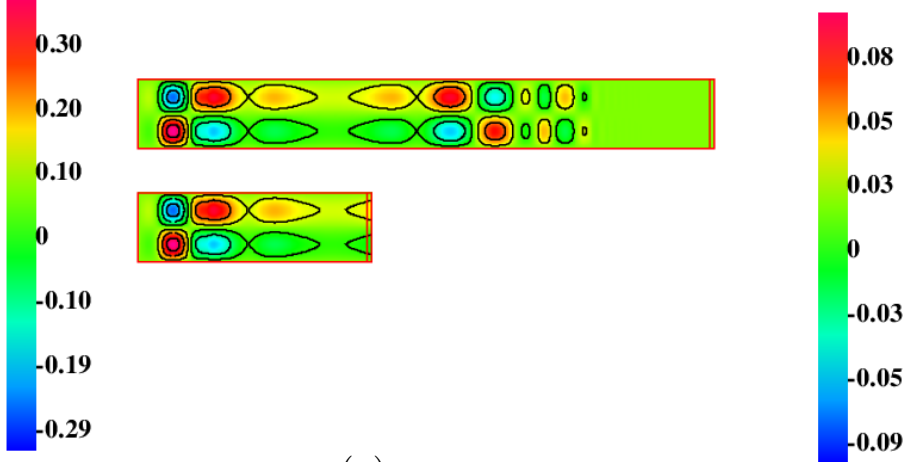

$(\mathrm{g})$
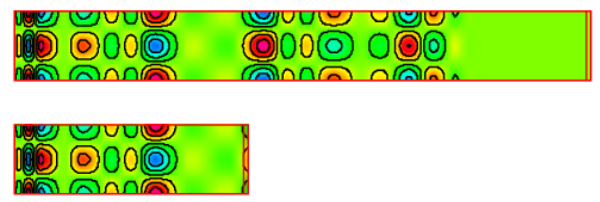

(h)

Figure 10: Pulse propagation in waveguide, material Type II: snapshots of solution: (a)-(b) $u_{x}$ and $u_{y}$ at $t=0 ;(\mathrm{c})-(\mathrm{d}) u_{x}$ and $u_{y}$ at $t=1$; (e)-(f) $u_{x}$ and $u_{y}$ at $t=3$; (g) $-(\mathrm{h}) u_{x}$ and $u_{y}$ at $t=6$. 

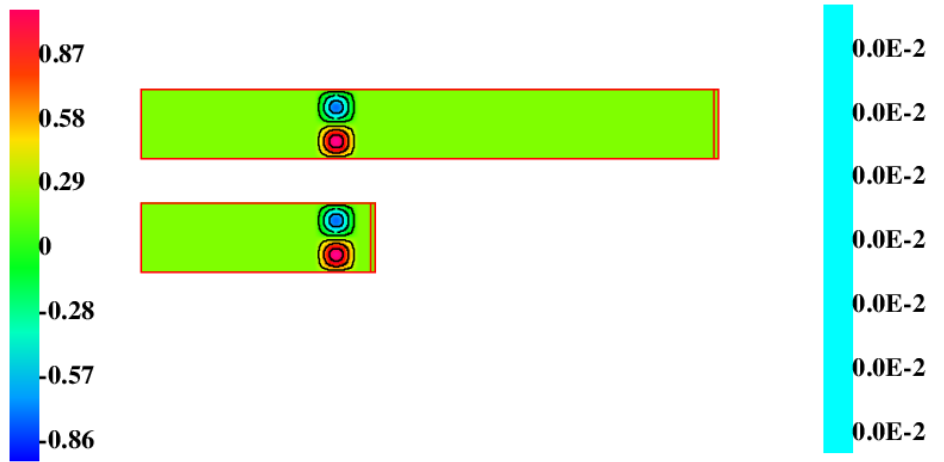

(a)

(b)
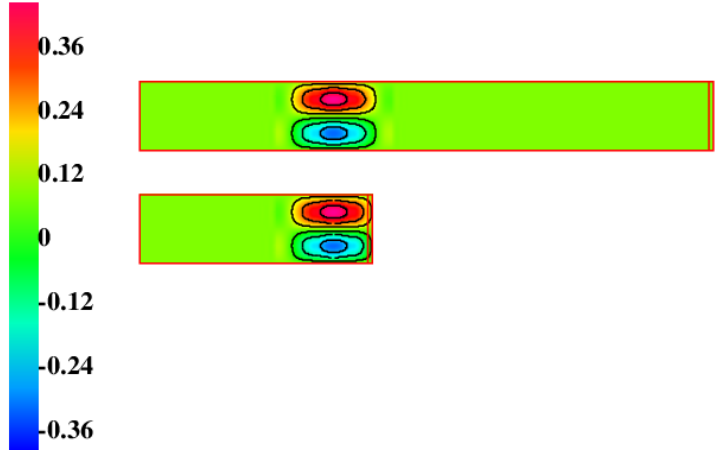

(1)

\section{.}

(c)
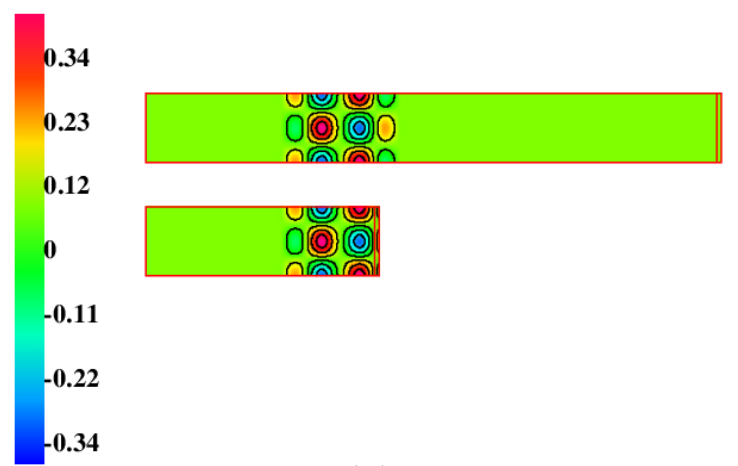

.11

2

.34

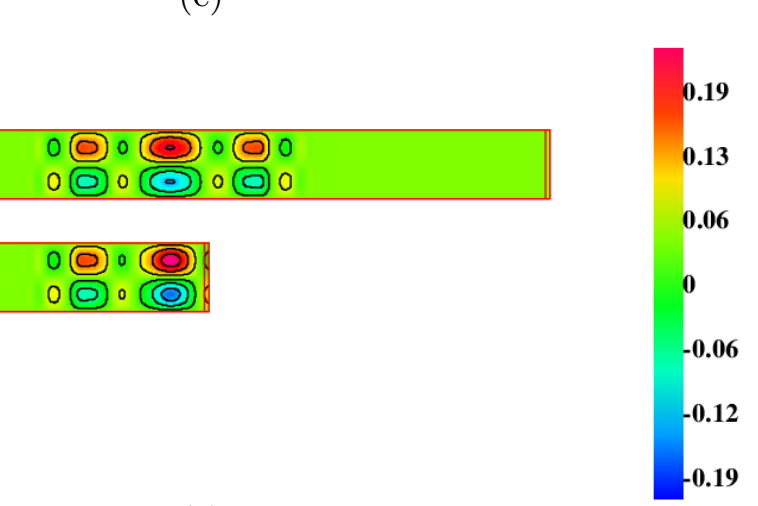

(e)

(f)
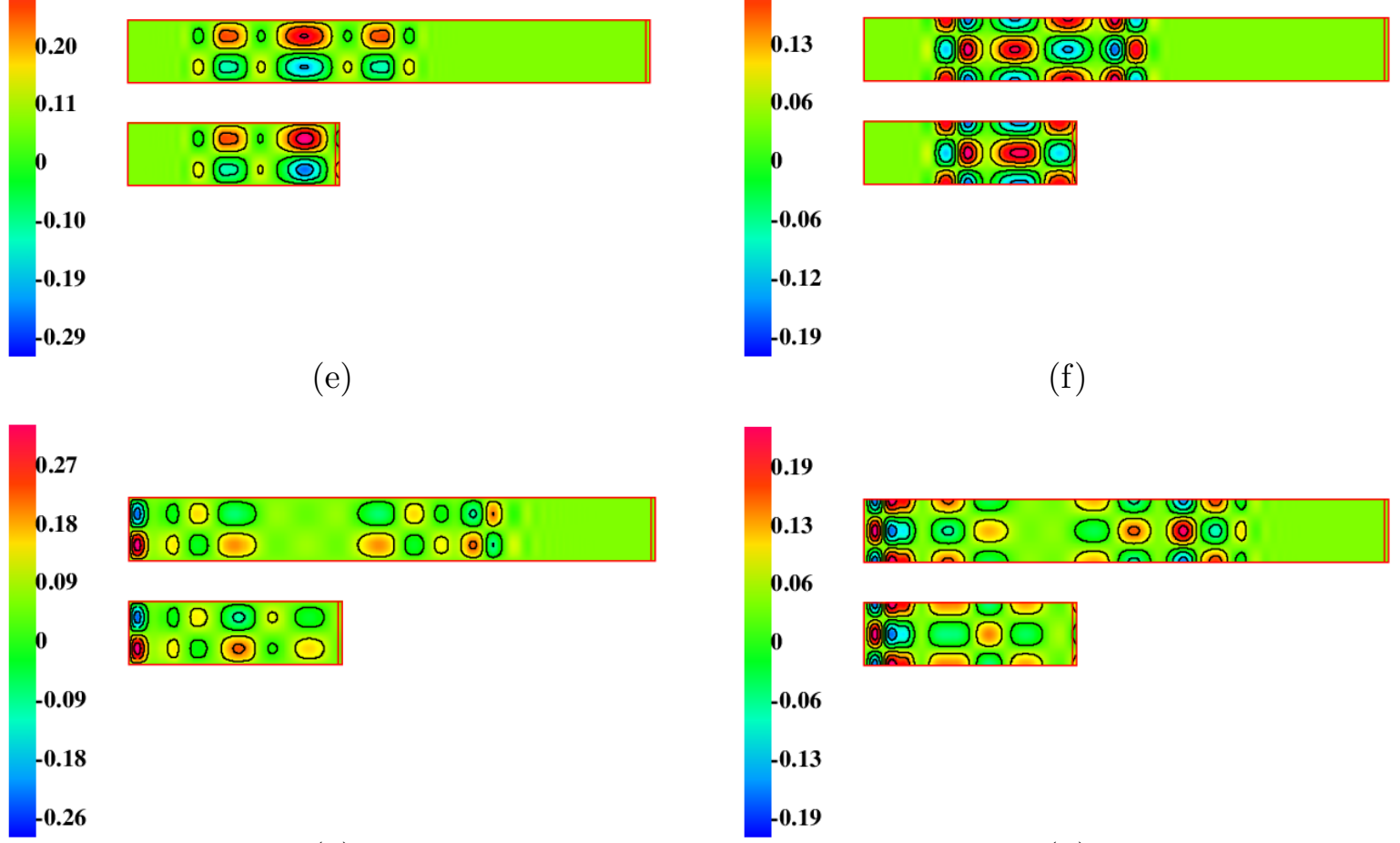

(d)

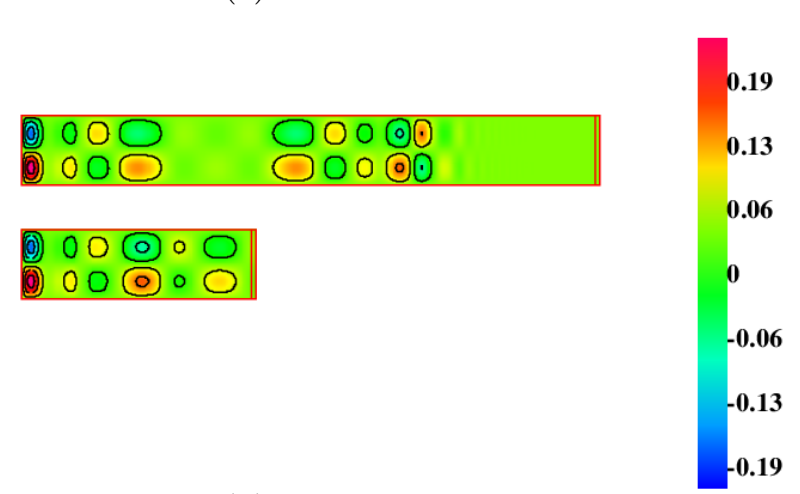

$(\mathrm{g})$
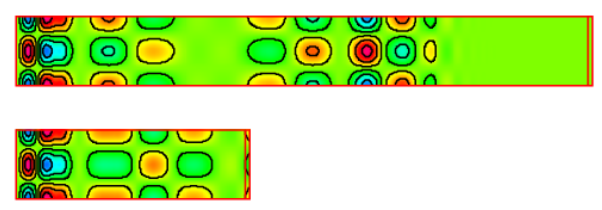

(h)

Figure 11: Pulse propagation in waveguide, material Type III: snapshots of solution: (a)-(b) $u_{x}$ and $u_{y}$ at $t=0 ;(\mathrm{c})-(\mathrm{d}) u_{x}$ and $u_{y}$ at $t=1 ;(\mathrm{e})-(\mathrm{f}) u_{x}$ and $u_{y}$ at $t=3 ;(\mathrm{g})-(\mathrm{h}) u_{x}$ and $u_{y}$ at $t=6$. 


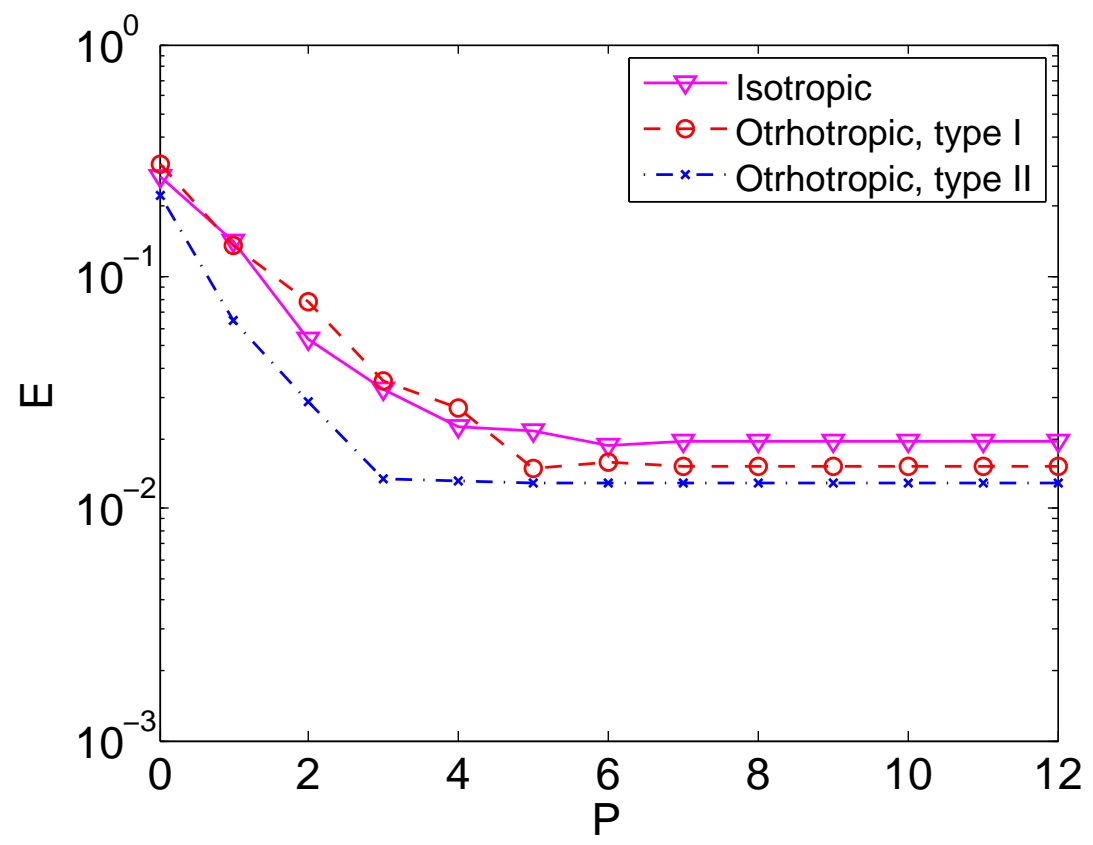

Figure 12: Accuracy of the stable DAB scheme for periodic boundary conditions, Mesh B

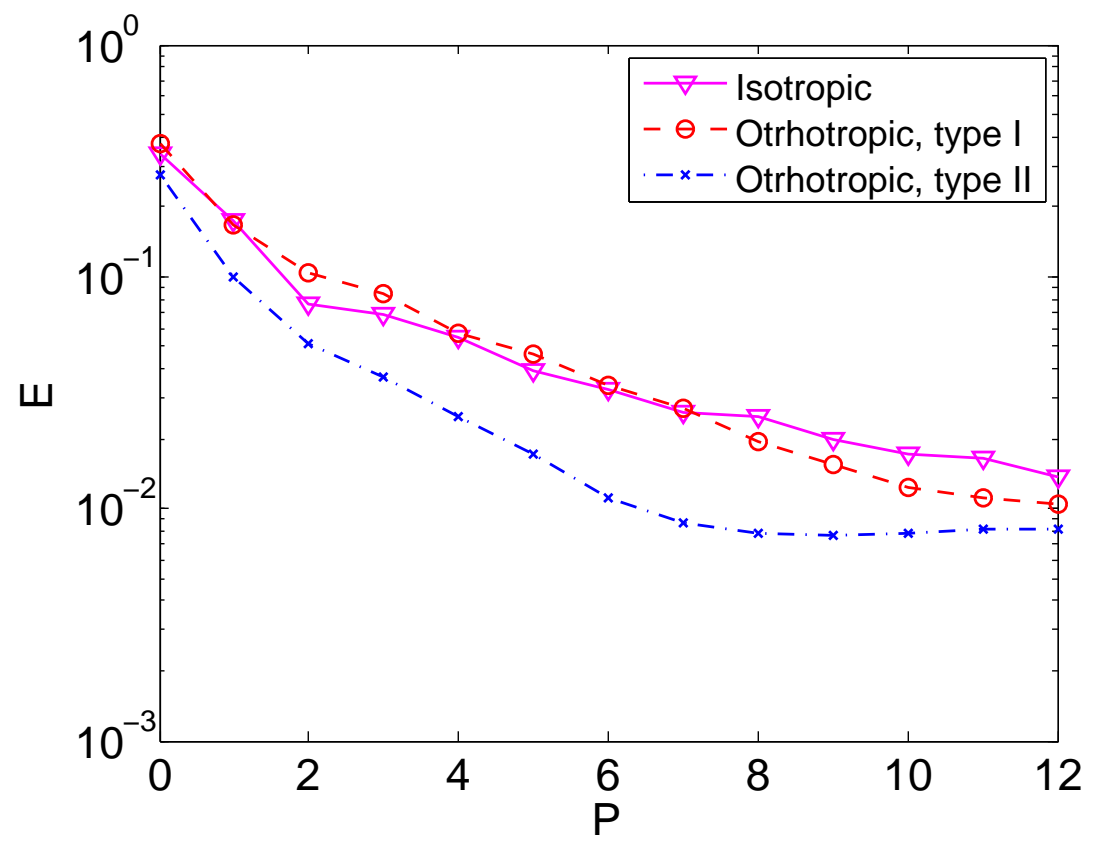

Figure 13: Accuracy of the stable DAB scheme for periodic boundary conditions, Mesh A, $T=20$ 


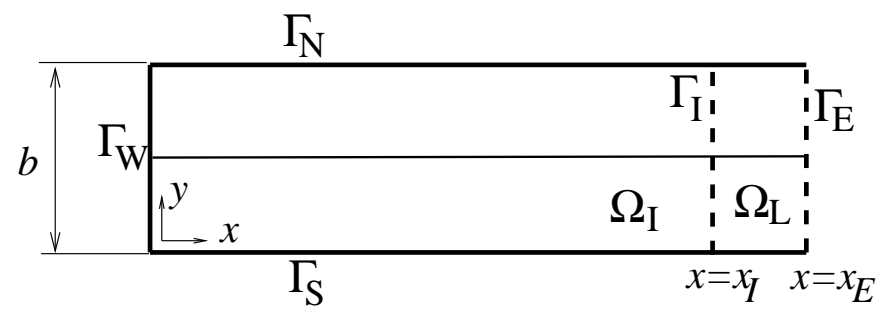

Figure 14: A truncated waveguide with longitudinal layers.

DAB parameter $P$, we repeated again the initial pulse run, this time with $T=20$. The results are shown in Fig. 13.

In this case the errors obtained are slightly larger than in the run with shorter simulation time, but more importantly, in can be seen that the decay of the error with $P$ is slower in all materials. This suggests that in order to obtain a desired accuracy for longer simulations, higher values of $P$ or a better choice of parameters is required.

\subsection{Initial pulse in two-layer waveguide}

In this example the geometry and the initial and boundary conditions are similar to the ones used in the single-layer example above. In the present case, however, we divide the waveguide and the DAB zone parallel to the $x$ axis into two layers of equal width, with different material properties. Thus, for all elements with centroids at $y_{c}>1.5$ we specify the isotropic material, while for the elements with centroids with $y_{c} \leq 1.5$ we prescribe either the material of Type I or Type II, see Fig. 14.

The procedure of separating the waveguide into two longitudinal layers was carried out both for the solved waveguide and for the reference waveguide. We took $\rho=1$ everywhere, and used the components of the elastic tensor that correspond to the definition of the materials of Type I and Type II, as given above. The obtained accuracy in this case is illustrated by the error plot given in Fig. 15. The error in the case of the isotropic/Type I domain is very similar in form to the domain comprised fully of a Type I material (see (6)), where the values of the error are only sightly larger than in the homogeneous case throughout the range of $P$. In the isotropic/Type II domain the decrease in the error is more gradual than in the comprised fully of a Type II material, since the former is dominated by the decrease in error in the isotropic material, which exhibits a slower decrease.

In Fig. 16 we show snapshots of the solution for the two-layer waveguide with an upper isotropic material and a lower Type I material.

One can see that in the case of the layered materials the pattern in the waveguide does not remain antisymmetric, however there is little inter-penetration of the contour structures between the layers during the simulation time. Additional experiments with various combinations of layers made of materials I, II and the isotropic material, not shown here for the sake of brevity, also give accurate solutions, which allow one to investigate interesting wave phenomena in layered anisotropic media. 


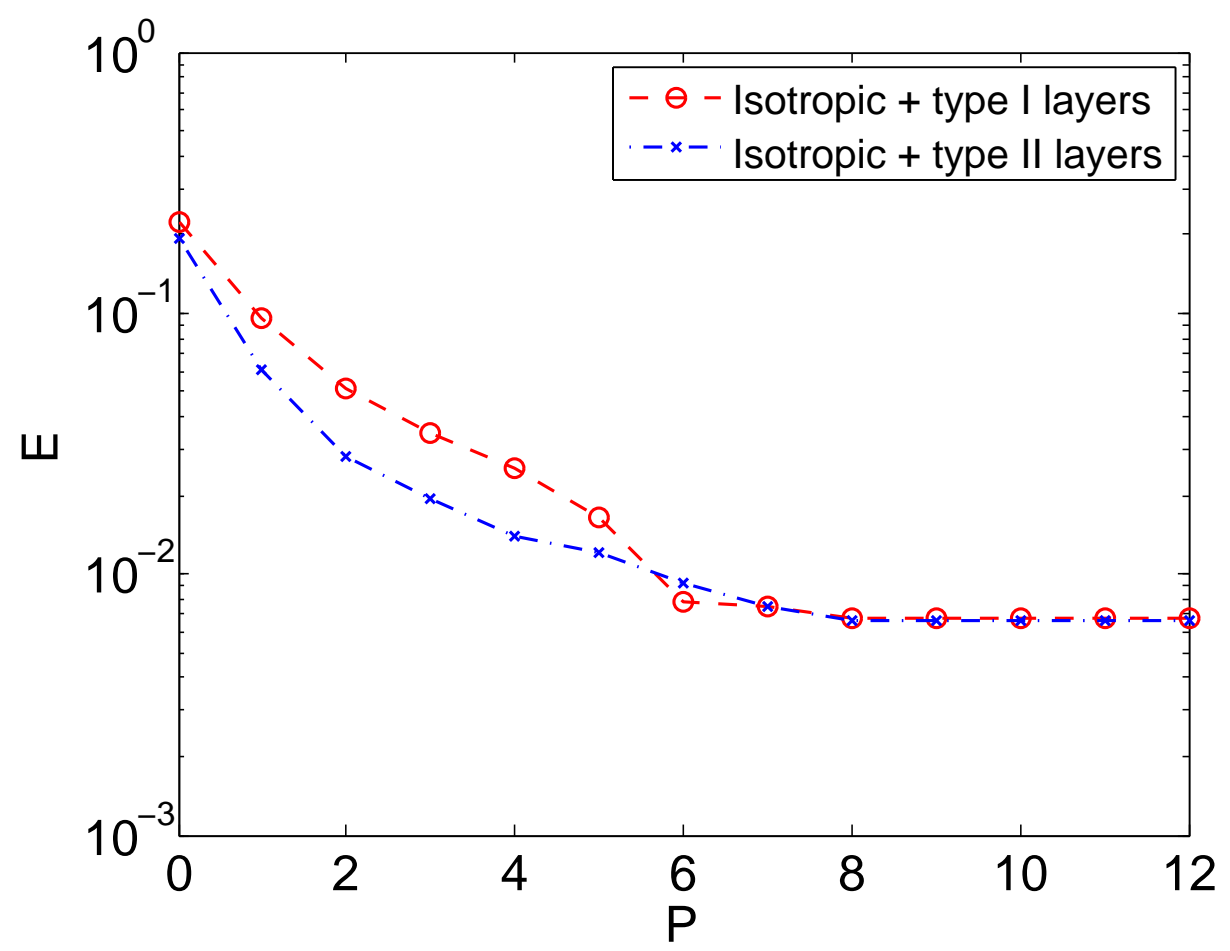

Figure 15: Accuracy of the two-layer (isotropic/orthotropic) DAB waveguide case

\section{Concluding Remarks}

In this paper the Double Absorbing Boundary (DAB) method for solving unbounded domain problems was applied to elastodynamics problems in anisotropic media. This method shares some of the properties of using a high-order ABC on a single boundary and of PML, but has some relative advantages with respect to both.

Some stability issues still need to be resolved. In particular, the following difficulties have to be addressed:

- Periodic boundary conditions were used in this work along the boundaries perpendicular to the DAB layer. When these were replaced by some physical conditions, like traction-free conditions, numerical stability was lost. It seems that this difficulty, which is currently under investigation, is not associated with the original DAB formulation at the continuous level, but with the semi-discrete formulation. The damped Newmrak scheme regains stability at the fully discrete level in the case of periodic boundary conditions, but not in some other cases.

- The difficulty of the stability of the DAB in the presence of inverse modes needs resolving. This is an open problem today. As mentioned in the Introduction, it is a 

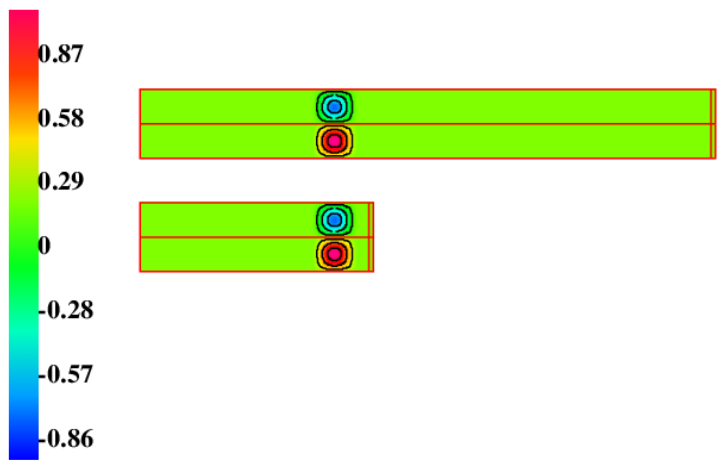

(a)
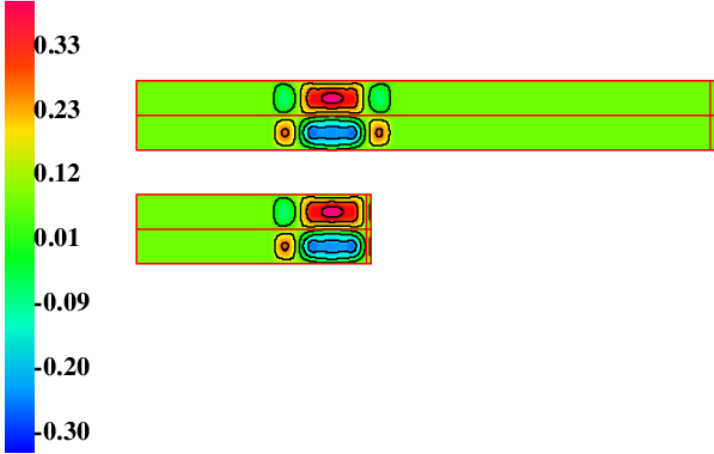

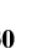

(c)
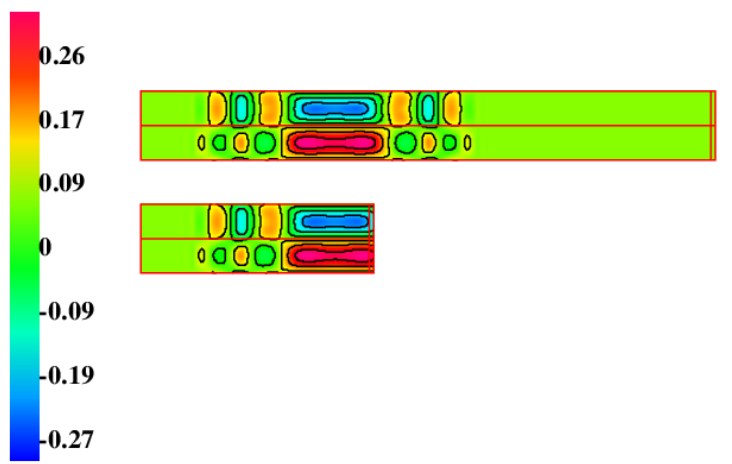

(e)
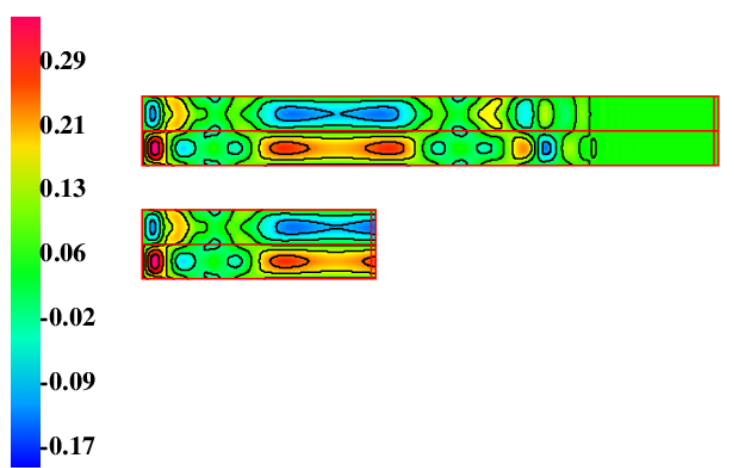

(g)

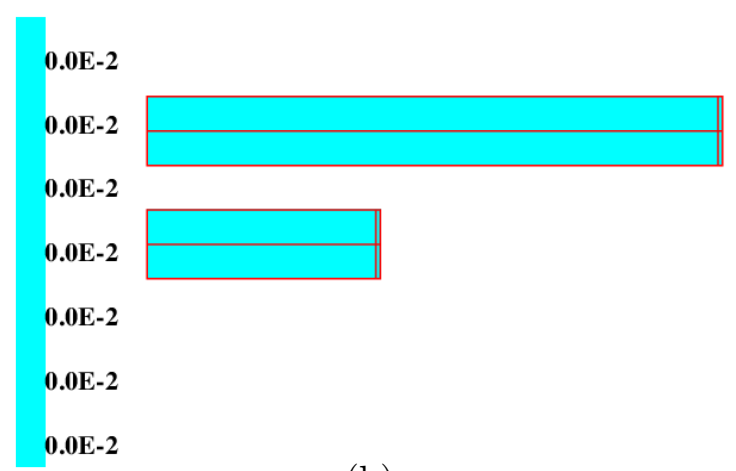

(b)
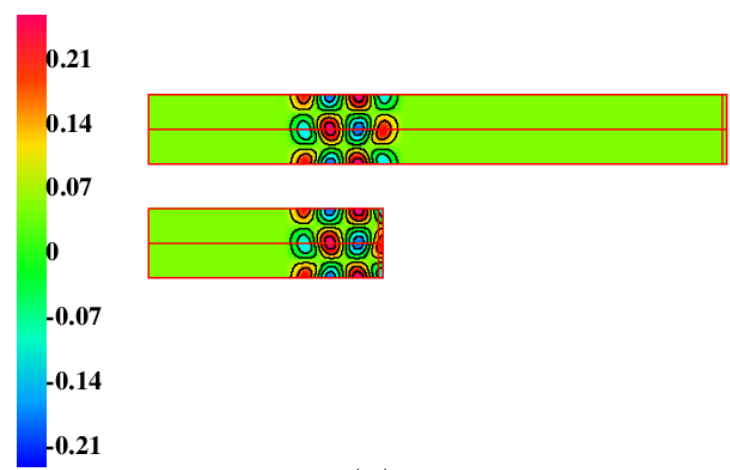

(d)


(f)
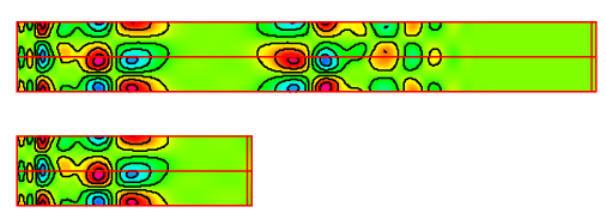

$\mathbf{- 0 . 0 5}$

$-\mathbf{0 . 1 1}$

$-\mathbf{0 . 1 5}$

(h)

Figure 16: Pulse propagation in waveguide, material Type I: snapshots of solution: (a)-(b) $u_{x}$ and $u_{y}$ at $t=0 ;(\mathrm{c})-(\mathrm{d}) u_{x}$ and $u_{y}$ at $t=1 ;(\mathrm{e})-(\mathrm{f}) u_{x}$ and $u_{y}$ at $t=3 ;(\mathrm{g})-(\mathrm{h}) u_{x}$ and $u_{y}$ at $t=6$. 
common difficulty to PMLs and to high order ABCs. In the case of PML, stabilized formulations exist $[28,29]$, but only with the price of losing the perfect matching.

\section{Acknowledgments}

This work was supported by the US-Israel Binational Science Foundation (BSF), grant number 890020 (Technion number 2011303). The work of DG was also supported by the fund provided through the Lawrence and Marie Feldman Chair in Engineering, and that of TH by NSF grant DMS-1418871. Any conclusions or recommendations in the paper are those of the authors and do not necessarily represent the views of the BSF or NSF.

\section{References}

[1] T. Hagstrom, "Radiation Boundary Conditions for the Numerical Simulation of Waves," Acta Numerica, 8, 47-106, 1999.

[2] D. Givoli, "High-Order Local Non-Reflecting Boundary Conditions: A Review," Wave Motion, 39, 319-326, 2004.

[3] D. Givoli, "Computational Absorbing Boundaries," in Computational Acoustics of Noise Propagation in Fluids, S. Marburg and B. Nolte, eds., Chapter 5, pp. 145-166, Springer, Berlin, 2008.

[4] A. Bermudez, L. Hervella-Nieto, A. Prieto and R. Rodriguez, "Perfectly Matched Layers for Time-Harmonic Second Order Elliptic Problems," Archives Comput. Meth. in Engng., 17, 77-107, 2010.

[5] F. Collino, "High Order Absorbing Boundary Conditions for Wave Propagation Models. Straight Line Boundary and Corner Cases," in Proc. 2nd Int. Conf. on Mathematical \& Numerical Aspects of Wave Propagation, R. Kleinman et al., Eds., SIAM, Delaware, pp. 161-171, 1993.

[6] B. Engquist and A. Majda, "Radiation Boundary Conditions for Acoustic and Elastic Wave Calculations," Comm. Pure Appl. Math., 32, 313-357, 1979.

[7] A. Bayliss and E. Turkel, "Radiation Boundary Conditions for Wave-Like Equations", Comm. Pure Appl. Math. 33 (1980) 707-725.

[8] J.P. Bérenger, "A Perfectly Matched Layer for the Absorption of Electromagnetic Waves," J. Comput. Phys., 114, 185-200, 1994.

[9] D. Appelö, T. Hagstrom and G. Kreiss, "Perfectly Matched Layers for Hyperbolic Systems: General Formulation, Well-posedness and Stability," SIAM J. Appl. Math., 67, 1-23, 2006. 
[10] D. Rabinovich, D. Givoli, J. Bielak and T. Hagstrom, "A Finite Element Scheme with a High Order Absorbing Boundary Condition for Elastodynamics," Comput. Meth. Appl. Mech. Engng., 200, 2048-2066, 2011.

[11] D. Rabinovich, D. Givoli and E. Bécache, "Comparison of High-order Absorbing Boundary Conditions and Perfectly Matched Layers in the Frequency Domain, Int. J. Num. Meth. in Biomed. Engng. (Formerly Commun. Numer. Meth. Engng.), 26, 1351-1369, 2010.

[12] G. Lancioni, "Numerical Comparison of High-order Absorbing Boundary Conditions and Perfectly Matched Layers for a Dispersive One-dimensional Medium," Comput. Meth. Appl. Mech. Engng., 209, 74-86, 2012.

[13] T. Hagstrom, D. Givoli, D. Rabinovich and J. Bielak, "The Double Absorbing Boundary Method," J. Comput. Phys., 259, 220-241, 2014.

[14] D. Baffet, T. Hagstrom and D. Givoli, "Double Absorbing Boundary Formulations for Acoustics and Elastodynamics," SIAM J. on Sci. Comput., 36, A1277-A1312, 2014.

[15] D. Rabinovich, D. Givoli, J. Bielak and T. Hagstrom, "The Double Absorbing Boundary Method for Elastodynamics in Homogeneous and Layered Media," Adv. Modeling and Simulation in Eng. Sci., 2:3, 2015.

[16] T. Hagstrom and T. Warburton, "A New Auxiliary Variable Formulation of HighOrder Local Radiation Boundary Conditions: Corner Compatibility Conditions and Extensions to First Order Systems," Wave Motion, 39, 327-338, 2004.

[17] T. Hagstrom and T. Warburton, "Complete Radiation Boundary Conditions: Minimizing the Long Time Error Growth of Local Methods", SIAM J. Numer. Anal., 47, 3678-3704, 2009.

[18] J. Lagrone and T. Hagstrom, "Double Absorbing Boundaries for Finite-Difference TimeDomain Electromagnetics", in review, 2016.

[19] D. Baffet, J. Bielak, D. Givoli, T. Hagstrom and D. Rabinovich, "Long-Time Stable High-Order Absorbing Boundary Conditions for Elastodynamics," Comput. Meth. Appl. Mech. Engng., 241-244, 20-37, 2012.

[20] D. Rabinovich, D. Givoli, T. Hagstrom and J. Bielak, "Stress-Velocity Complete Radiation Boundary Conditions," J. Comput. Acoust., 21, 1350003-1-38, 2013.

[21] J. Lysmer and R.L. Kuhlemeyer, "Finite Dynamic Model for Infinite Media," J. Eng. Mech. Div. ASCE, 95, 859-877, 1969.

[22] A. Bamberger, B. Chalindar, P. Joly, J.E. Roberts and J.L. Teron, “Absorbing Boundary Conditions for Rayleigh Waves," SAIM J. Sci. Stat. Comput., 9, 1016-1049, 1988. 
[23] H.S. Bao, J. Bielak, O. Ghattas, L.F. Kallivokas, D.R. O'Hallaron, J.R. Shewchuk and J.F. Xu, "Large-Scale Simulation of Elastic Wave Propagation in Heterogeneous Media on Parallel Computers," Comput. Meth. Appl. Mech. Engng., 152, 85-102, 1998.

[24] J. Bielak, O. Ghattas and E.J. Kim, "Parallel Octree-Based Finite Element Method for Large-Scale Earthquake Ground Motion Simulation," Comput. Model. Engng. Sci., 10, 99-112, 2005.

[25] S.M. Day, R. Graves, J. Bielak, D. Dreger, S. Larsen, K.B. Olsen, A. Pitarka and L. Ramirez-Guzman, "Model for Basin Effects on Long-Period Response Spectra in Southern California," Earthquake Spectra, 24, 257-277, 2008.

[26] K. Duru and G. Kreiss, "Numerical Interaction of Boundary Waves with Perfectly Matched Layers in Two Space Dimensional Elastic Waveguides," Wave Motion, 51, 445-465, 2014.

[27] E. Bécache, S. Fauqueux and P. Joly, "Stability of Perfectly Matched Layers, Group Velocities and Anisotropic Waves," J. Comput. Phys., 188, 399-433, 2003.

[28] K.C. Meza-Fajardo and A.S. Papageorgiou, "A Nonconvolutional, Split-Field, Perfectly Matched Layer for Wave Propagation in Isotropic and Anisotropic Elastic Media: Stability Analysis," Bull. Seism. Soc. Am., 98, 1811-1836, 2008.

[29] R. Martin, D. Komatitsch and S.D. Gedney, "A Variational Formulation of a Stabilized Unsplit Convolutional Perfectly Matched Layer for The Isotropic or Anisotropic Seismic Wave Equation," Comp. Model. Engn. Sci., 37, 274-304, 2008.

[30] F. Collino and C. Tsogka, "Application of the Perfectly Matched Absorbing Layer Model to the Linear Elastodynamic Problem in Anisotropic Heterogeneous Media," Geophys., 66, 294-307, 2001.

[31] D.S. Bindel and S. Govindjee, "Elastic PMLs for Resonator Anchor Loss Simulations," Int. J. Numer. Meth. Engng., 64, 789-818, 2005.

[32] I. Harari and U. Albocher, "Studies of FE/PML for Exterior Problems of TimeHarmonic Elastic Waves," Comput. Meth. Appl. Mech. Engng., 195, 3854-3879, 2006.

[33] J.M. de Oliveira Barbosa, J. Park and E. Kausel, "Perfectly Matched Layers in the Thin Layer Method," Comput. Meth. Appl. Mech. Engng., 217, 262-274, 2012.

[34] E. Skelton, S. Adams and R. Craster, "Guided elastic waves and perfectly matched layers", Wave Motion, 44, 573-592, 2007.

[35] E. Bécache, D. Givoli and T. Hagstrom, "High Order Absorbing Boundary Conditions for Anisotropic and Convective Wave Equations," J. Comput. Phys., 229, 1099-1129, 2010. 
[36] G. Kreiss and K. Duru, "Discrete Stability of Perfectly Matched Layers for Anisotropic Wave Equations in First and Second Order Formulation," BIT Num. Math., 53, 641$663,2013$.

[37] L.D. Landau and E.M. Lifshitz, Theorey of Elasticity, 3rd Ed., Pergamon Press, Exeter, UK, 1986.

[38] R.L. Higdon, "Radiation Boundary Conditions for Elastic Wave Propagation," SIAM J. Numer. Anal., 27, N4, 831-870, 1990.

[39] D. Rabinovich, D. Givoli, J. Bielak and T. Hagstrom, "A finite Element Scheme with a High Order Absorbing Boundary Condition for Elastodynamics," Comput. Meth. Appl. Mech. Engng., 200, 2048-2066, 2011.

[40] D. Givoli, T. Hagstrom and I. Patlashenko, "Finite Element Formulation with High Order Absorbing Boundary Conditions for Time-Dependent Waves," Comput. Meth. Appl. Mech. Engng., 195, 3666-3690, 2006.

[41] D. Givoli and B. Neta, "High-Order Non-Reflecting Boundary Scheme for TimeDependent Waves," J. Comput. Phys., 186, 24-46, 2003.

[42] T.J.R. Hughes, The Finite Element Method, Prentice Hall, Englewood Cliffs, N.J., 1987. 